\title{
Alternating Sign Matrices and Hypermatrices, and a Generalization of Latin Squares
}

\author{
Richard A. Brualdi* \\ Geir Dahl ${ }^{\dagger}$ \\ December 8, 2017
}

\section{To appear in Advances in Applied Mathematics}

\begin{abstract}
An alternating sign matrix, or $\mathrm{ASM}$, is a $(0, \pm 1)$-matrix where the nonzero entries in each row and column alternate in sign. We generalize this notion to hypermatrices: an $n \times n \times n$ hypermatrix $A=\left[a_{i j k}\right]$ is an alternating sign hypermatrix, or ASHM, if each of its planes, obtained by fixing one of the three indices, is an ASM. Several results concerning ASHMs are shown, such as finding the maximum number of nonzeros of an $n \times n \times n$ ASHM, and properties related to Latin squares. Moreover, we investigate completion problems, in which one asks if a subhypermatrix can be completed (extended) into an ASHM. We show several theorems of this type.
\end{abstract}

Key words. Alternating sign matrix, hypermatrix, completion, Latin square. AMS subject classifications. 05B20, 15A69, 15B48.

\section{Introduction}

Let $A$ be an $n \times n(0, \pm 1)$-matrix. Then $A$ is an alternating sign matrix, abbreviated ASM, provided in each of the $2 n$ lines of $A$, that is, its rows and

${ }^{*}$ Department of Mathematics, University of Wisconsin, Madison, WI 53706, USA. brualdi@math. wisc.edu

${ }^{\dagger}$ Department of Mathematics, University of Oslo, Norway. geird@math.uio.no 
columns, the nonzeros alternate beginning and ending with a +1 . Permutation matrices are ASMs without any -1's. ASMs were defined by Mills, Robbins, and Ramsey [13] and have a fascinating history which can be found in [2]. Extending some of the work reported in [1] and [15], we carried out in [4] a recent study of ASMs and related matrix classes and polyhedra where additional references, besides those given here, can be found. Our goal is to generalize ASMs to three-dimensional matrices called hypermatrices. In doing so, we were led to a fascinating generalization of classical Latin squares.

Let $A=\left[a_{i j k}\right]$ be an $n \times n \times n$ hypermatrix. We refer to $i$ as the row index, $j$ as the column index, and $k$ as the vertical index of the hypermatrix $A$. Then $A$ has three types of lines, each of cardinality $n$ :

(i) The row lines (variable row index) $A_{* j k}=\left[a_{i j k}: i=1,2, \ldots n\right], \quad(1 \leq$ $j, k \leq n)$;

(ii) The column lines (variable column index) $A_{i * k}=\left[a_{i j k}: j=1,2, \ldots n\right],(1 \leq$ $i, k \leq n)$;

(iii) The vertical lines (variable vertical index) $A_{i j *}=\left[a_{i j k}: k=1,2, \ldots n\right],(1 \leq$ $i, j \leq n)$.

Similarly, $A$ has three types of planes, each of cardinality $n^{2}$ :

(i) The horizontal planes (or row-column planes) (variable row and column indices) $A_{k}^{\mathrm{h}}=A_{* * k}=\left[a_{i j k}: i, j=1,2, \ldots n\right],(1 \leq k \leq n)$;

(ii) The row-vertical planes (variable row and vertical indices) $A_{j}^{\mathrm{cv}}=A_{* j *}=$ $\left[a_{i j k}: i, k=1,2, \ldots n\right],(1 \leq j \leq n)$;

(iii) The column-vertical planes (variable column and vertical indices) $A_{i}^{\mathrm{rv}}=$ $A_{i * *}=\left[a_{i j k}: j, k=1,2, \ldots n\right],(1 \leq i \leq n)$.

The intersection of two planes of different types is a line; for instance, the intersection of a horizontal plane with a column-vertical plane is a column line:

$$
A_{* * k} \cap A_{i * *}=A_{i * k} .
$$

We usually denote the $n \times n \times n$ hypermatrix $A$ by

$$
A=\left[A_{1}^{\mathrm{h}}, A_{2}^{\mathrm{h}}, \ldots, A_{n}^{\mathrm{h}}\right], \text { abbreviated to } A=\left[A_{1}, A_{2}, \ldots, A_{n}\right]
$$


where the $A_{i}$ are the horizontal planes $A_{* * k}$. To denote the fact that $A$ is a 3-dimensional array, we also write

$$
A=A_{1} \nearrow A_{2} \nearrow \ldots \nearrow A_{n}
$$

where the north-east arrow $A_{i} \nearrow A_{i+1}$ is read as $A_{i}$ is below $A_{i+1}$ (or $A_{i+1}$ is on top of $A_{i}$ ). We can also write

$$
A=\left[A_{1}^{\mathrm{cv}}, A_{2}^{\mathrm{cv}}, \ldots, A_{n}^{\mathrm{cv}}\right] \text { and } A=\left[A_{1}^{\mathrm{rv}}, A_{2}^{\mathrm{rv}}, \ldots, A_{n}^{\mathrm{rv}}\right] .
$$

We can generalize an alternating sign matrix to an $n \times n \times n(0, \pm 1)$ hypermatrix $A$ in two natural ways:

(a) $A$ is an alternating sign hypermatrix, abbreviated ASHM, provided in each of its $3 n^{2}$ lines, the nonzeros alternate beginning and ending with $\mathrm{a}+1$, equivalently, each of its $3 n$ planes is an ASM.

(b) $A$ is a planar alternating sign hypermatrix, abbreviated PASHM, provided each of its $n \times n$ horizontal planes is an ASM and each of its vertical lines sums to 1 . (We can replace horizontal planes with any of the other two types of planes and vertical lines with the appropriate lines.)

The property of being a PASHM is weaker than being a ASHM. If in our definition of a PASHM we replace the condition that the vertical lines sum to 1 with the condition that the nonzeros in the vertical lines alternate beginning and ending with a +1 , then the result would be an ASHM. The weaker condition that the vertical lines sum to 1 connects the horizontalplane ASMs $A_{1}, A_{2}, \ldots, A_{n}$ with one another.

If $\left[A_{1}, A_{2}, \ldots, A_{n}\right]$ is an ASHM (respectively, a PASHM) then so is the hypermatrix $\left[A_{n}, \ldots, A_{2}, A_{1}\right]$ obtained by reversing the order of its horizontal planes. It follows that for $i \leq n / 2$, the ASMs $A_{i}$ and $A_{n-i}$ satisfy similar properties. More generally, the set of $n \times n \times n$ ASHMs is invariant under the symmetry group of the unit cube. This group, called the hyperoctahedral group $^{1}$, is the direct product $\mathcal{S}_{2} \times \mathcal{S}_{4}$ of the symmetric permutation groups $\mathcal{S}_{2}$ and $\mathcal{S}_{4}$ and has size 48 .

An $n \times n \times n$ permutation hypermatrix is a $(0,1)$-hypermatrix with exactly one 1 in each line, and these are the ASHMs not having any -1 's. If $A=$

\footnotetext{
${ }^{1}$ It is also the group of signed permutations $\pi$ of $\{ \pm 1, \pm 2, \pm 3, \pm 4\}$ satisfying $\pi(-i)=$ $-\pi(i)$ for all $i$.
} 
$\left[A_{1}, A_{2}, \ldots, A_{n}\right]$ is a PASHM, then $A_{1}$ and $A_{n}$ and, in fact, each of the first and last planes of each of the three types, must be permutation matrices. Thus, thinking of $A$ as an $n \times n \times n$ cube, the six boundary facets of the cube are $n \times n$ permutation matrices.

We remark that the question of generalizing the Birkhoff - von Neumann theorem (which asserts that the extreme points of the polytope of $n \times n$ doubly stochastic matrices are the $n \times n$ permutation matrices) to tensors (that is, hypermatrices) is discussed in [10] (see also, more recently, [11]). It was shown that the polytope of multistochastic hypermatrices in general has other vertices than those corresponding to permutation hypermatrices.

Example 1. The following is a $3 \times 3 \times 3$ ASHM:

$$
L_{3}:=\left[\begin{array}{lll}
1 & 0 & 0 \\
0 & 1 & 0 \\
0 & 0 & 1
\end{array}\right] \nearrow\left[\begin{array}{rrr}
0 & 1 & 0 \\
1 & -1 & 1 \\
0 & 1 & 0
\end{array}\right] \nearrow\left[\begin{array}{lll}
0 & 0 & 1 \\
0 & 1 & 0 \\
1 & 0 & 0
\end{array}\right] .
$$

We get another ASHM by interchanging the top and bottom planes of $L_{3}$. It is easy to verify that every $3 \times 3 \times 3$ PASHM is an ASHM. An example of an PASHM which is not an ASHM is

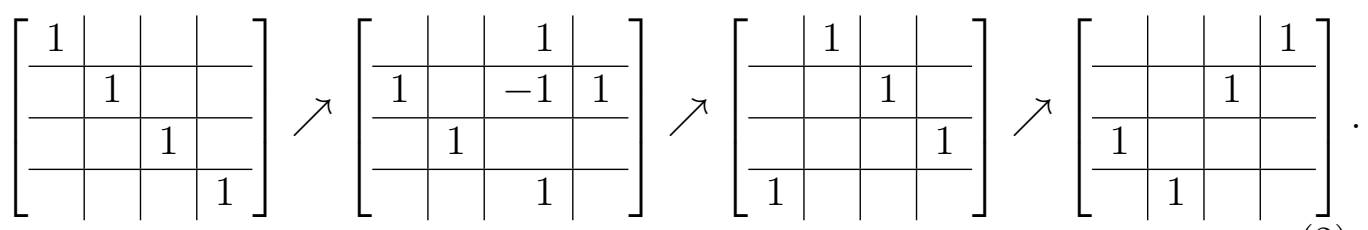

An $n \times n \times n$ permutation hypermatrix $A=\left[a_{i j k}\right]$ is "equivalent" to an $n \times n$ Latin square (sometimes abbreviated as LS) $L(A)=\left[l_{i j}\right]$. This equivalence results by setting $l_{i j}=k$ if and only if $a_{i j k}=1$.

For example, the $3 \times 3 \times 3$ permutation hypermatrix

$$
\left[\begin{array}{lll}
1 & 0 & 0 \\
0 & 1 & 0 \\
0 & 0 & 1
\end{array}\right] \nearrow\left[\begin{array}{lll}
0 & 1 & 0 \\
0 & 0 & 1 \\
1 & 0 & 0
\end{array}\right] \nearrow\left[\begin{array}{lll}
0 & 0 & 1 \\
1 & 0 & 0 \\
0 & 1 & 0
\end{array}\right]
$$

gives the Latin square

$$
\left[\begin{array}{lll}
1 & 2 & 3 \\
3 & 1 & 2 \\
2 & 3 & 1
\end{array}\right]
$$


Another way to view this equivalence is as follows. Consider the $n \times n$ matrix $J_{n}$ of all 1's and a decomposition of $J_{n}$ into $n$ permutation matrices

$$
J_{n}=P_{1}+P_{2}+\cdots+P_{n} .
$$

Then $P_{1} \nearrow P_{2} \nearrow \ldots \nearrow P_{n}$ is an $n \times n \times n$ permutation hypermatrix and

$$
1 P_{1}+2 P_{2}+\cdots+n P_{n}
$$

is a Latin square; every $n \times n \times n$ permutation hypermatrix and every $n \times n$ Latin square arises in this way.

Let $A=\left[A_{1}, A_{2}, \ldots, A_{n}\right]$ be an $n \times n \times n$ PASHM where, therefore, $A_{1}, A_{2}, \ldots, A_{n}$ are $n \times n$ ASMs. Since all vertical line sums of $A$ equal 1 , it follows that

$$
J_{n}=A_{1}+A_{2}+\cdots+A_{n}
$$

Let

$$
L(A)=1 A_{1}+2 A_{2}+\cdots+n A_{n} .
$$

Then in view of the above discussion, we call $L(A)$ a PASHM Latin-like square, shortened to PASHL. If $A$ is an ASHM, we call $L(A)$ an ASHM Latinlike square, shortened to ASHL. Thus $n \times n$ PASHML's result from certain decompositions of $J_{n}$ into $n n \times n$ ASMs. Ordinary Latin squares result in this way when $A$ is a permutation hypermatrix, that is, when $A_{1}, A_{2}, \ldots, A_{n}$ are $n \times n$ permutation matrices. So $n \times n \times n$ ASHMs and PASHMs can themselves be regarded as generalizations of $n \times n$ Latin squares.

Problems concering hypermatrices tend to be more difficult than similar problems for matrices. As an example of this, consider the classical König's minmax theorem for $(0,1)$-matrices $([8])$, which asserts that the term rank of a matrix $A$ (defined as the maximum number of nonzeros in $A$, no two in the same line) equals the minimum number of lines needed to cover all the nonzeros in $A$. The term rank may be found in polynomial time by computing a maximum matching in the corresponding bipartite graph. Now, for an $n \times n \times n$ hypermatrix $A$, define the term rank of $A$ as the maximum number of nonzeros in $A$, no two of which are on a common line.

Theorem 2. Finding the term rank of a hypermatrix is NP-hard.

Proof. This follows from the fact that the 3-dimensional matching problem, denoted by 3D-MATCH, is NP-hard ([12]). In 3D-MATCH there are given 
three disjoint sets $I, J$ and $K$, each of cardinality $n$, and a set $S \subseteq I \times J \times K$. A subset $M \subseteq S$ is a 3-dimensional matching if any two distinct elements $(i, j, k)$ and $\left(i^{\prime}, j^{\prime}, k^{\prime}\right)$ in $M$ satisfy $i \neq i^{\prime}, j \neq j^{\prime}$ and $k \neq k^{\prime}$. Then 3D-MATCH asks for the largest size of a 3 -dimensional matching. This problem reduces to finding the term rank in the $n \times n \times n$ hypermatrix $A=\left[a_{i j k}\right]$ defined by $a_{i j k}=1$ whenever $(i, j, k) \in S$. Therefore, computing the term rank of a hypermatrix is NP-hard.

\section{Diamond ASHMs}

A convex ASM (called dense in [9]) is defined to be an ASM having the property that there are not any zeros between nonzeros in both rows and columns. There is a family $\mathcal{F}_{n}=\left\{F_{n}^{1}, F_{n}^{2}, \ldots, F_{n}^{n}\right\}$ of $n \times n$ convex ASMs where $F_{n}^{k}$ is defined in the following way: There are +1 's in the positions in the stripes of $F_{n}^{k}$ running from position $(1, k)$ to position $(k, 1)$, from $(k, 1)$ to position $(n, n-k+1)$, from $(n, n-k+1)$ to position $(n+1-k, n)$, and from $(n+1-k, n)$ back to position $(1, k)$. Each of the entries within the region bordered by these four stripes is nonzero and so is uniquely determined, and all positions outside of this region are zero. In particular, $F_{n}^{1}$ is the $n \times n$ identity matrix $I_{n}$ and $F_{n}^{n}$ is the permutation matrix $L_{n}$ with 1 's on the back diagonal (running from position $(1, n)$ to $(n, 1)$ ). For example,

$$
F_{6}^{3}=\left[\begin{array}{r|r|r|r|r|r} 
& & 1 & & & \\
\hline & 1 & -1 & 1 & & \\
\hline 1 & -1 & 1 & -1 & 1 & \\
\hline & 1 & -1 & 1 & -1 & 1 \\
\hline & & 1 & -1 & 1 & \\
\hline & & & 1 & &
\end{array}\right] .
$$

If $n$ is odd, then $F_{n}^{(n+1) / 2}$ is the diamond $A S M$ of odd order; if $n$ is even, then $F_{n}^{n / 2}$ and $F_{n}^{(n+2) / 2}$ are the diamond $A S M s$ of even order. The ASM $F_{6}^{3}$ above is a diamond ASM.

For a $(0, \pm 1)$-matrix $B$, let $\sigma_{+}(B)$ equal the number of its +1 's and $\sigma_{-}(B)$ the number of its -1 's. Then $\sigma(B)=\sigma_{+}(B)+\sigma_{-}(B)$ is the number of nonzeros of $B$. If $2 k \leq n$, we have

$$
\sigma_{+}\left(F_{n}^{k}\right)=k(n-k+1) \text { and } \sigma_{-}\left(F_{n}^{k}\right)=(k-1)(n-k),
$$


and hence

$$
\sigma\left(F_{n}^{k}\right)=(n-k+1) k+(n-k)(k-1)=2 k(n-k+1)-n .
$$

The difference between the number of nonzeros of $F_{n}^{k+1}$ and those of $F_{n}^{k}$ equals $2(n-2 k)$ for $2 k<n$. The difference between the number of -1 's of $F_{n}^{k+1}$ and those of $F_{n}^{k}$ equals $(n-2 k)$ for $2 k<n$.

We define an ASHM to be convex provided that there are not any zeros between nonzeros in any of its lines. Some special $n \times n \times n$ convex ASHMs are the diamond $A S H M s$ defined by

$$
\mathfrak{D}_{n}=F_{n}^{1} \nearrow F_{n}^{2} \nearrow \cdots \nearrow F_{n}^{n} \text { and } F_{n}^{n} \nearrow F_{n}^{n-1} \nearrow \cdots \nearrow F_{n}^{1},
$$

both denoted by $\mathfrak{D}_{n}$ for convenience. It is easy to check that $\mathfrak{D}_{n}$ is indeed an ASHM.

Example 3. The diamond ASHM $\mathfrak{D}_{5}$ is given by

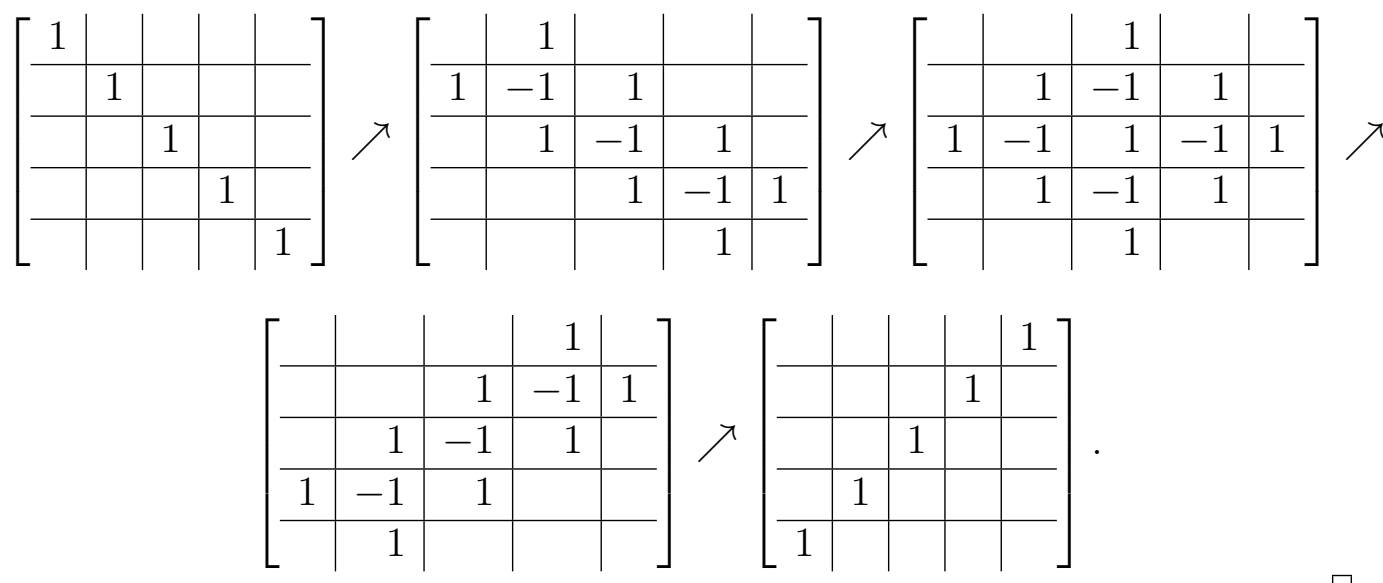

For each $k=1,2, \ldots, n$, the matrix $F_{n}^{1}+F_{n}^{2}+\cdots+F_{n}^{k}$ is an $n \times n$ $(0,1)$-matrix with exactly $k$ ones in each row and column; in particular, $F_{n}^{1}+F_{n}^{2}+\cdots+F_{n}^{k}$ has $n$ more ones than $F_{n}^{1}+F_{n}^{2}+\cdots+F_{n}^{k-1}$. In fact, this is a property shared by all $n \times n \times n$ ASHMs.

Lemma 4. Let $A=\left[A_{1}, A_{2}, \ldots, A_{n}\right]$ be an $A S H M$ and, for each $k=1,2, \ldots, n$, let $A^{(k)}=A_{1}+A_{2}+\cdots+A_{k}$. Then each $A^{(k)}$ is a $(0,1)$-matrix with $k$ ones in every row and column. In particular, $A^{(k+1)}$ has exactly $n$ more 1 's than $A^{(k)}$ for $k=1,2, \ldots, n-1$. 
Proof. Since $A$ is an ASHM, each $A^{(k)}$ is a $(0,1)$-matrix. Every line sum in $A_{i}$ is 1 , and therefore every line sum in $A^{(k)}=A_{1}+A_{2}+\cdots+A_{k}$ is $k$. So, $A^{(k)}$ has $k n$ ones, and the last statement follows.

The number of nonzeros in $\mathfrak{D}_{n}$ is computed to be

$$
\sum_{k=1}^{n}(2 k(n+1-k)-n)=\frac{n\left(n^{2}+2\right)}{3} .
$$

There are other ASHMs with the same number of nonzeros. For example, if $n=4$, the ASHM

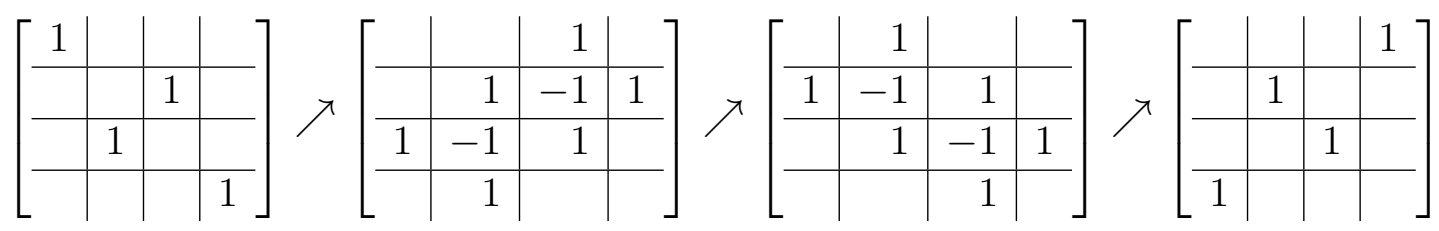

has the same number 24 of nonzeros as $\mathfrak{D}_{4}$.

In [5] it was shown that the $n \times n$ diamond ASMs have the largest number of nonzeros among all the $n \times n$ ASMs. In the next theorem we show the corresponding property for ASHMs. Recall that if $i \leq \frac{n+1}{2}$, the number of nonzeros in the $i$ th row (or column) of an $n \times n$ ASM is at most $2 i-1$. Since an ASM remains an ASM when the order of its rows are reversed (row $i$ becomes row $(n+1-i)$ for $1 \leq i \leq n)$ a similar inequality holds for rows $n, n-1, \ldots, \frac{n+1}{2}$. Equality holds in all these inequalities for diamond ASMs.

Consider an $n \times n \times n$ diamond ASHM $\mathfrak{D}_{n}=\left[F_{n}^{1}, F_{n}^{2}, \ldots, F_{n}^{n}\right]$. The ASMs $F_{n}^{1}, F_{n}^{2}, \ldots, F_{n}^{n}$ are the horizontal planes of $\mathfrak{D}_{n}$. The column-vertical planes of $\mathfrak{D}_{n}$ are also $F_{n}^{1}, F_{n}^{2}, \ldots, F_{n}^{n}$ as are the row-vertical planes. Thus, both give the diamond ASHM $\mathfrak{D}_{n}$. This symmetry of $\mathfrak{D}_{n}$ is the hypermatrix analogue of the symmetry of the diamond ASMs. The $i$ th row of the $k$ th horizontal plane $F_{n}^{k}$ is also the $k$ th row of the $i$ th row-vertical plane $F_{n}^{i}$ of $\mathfrak{D}_{n}$, and hence the number of its nonzeros is $2 \min \{i, k\}-1$.

Theorem 5. The maximum number $m_{n}$ of nonzeros in an $n \times n \times n A S H M$ is given by

$$
m_{n}=\frac{n\left(n^{2}+2\right)}{3}
$$

and this maximum is attained by the diamond $A S H M \mathfrak{D}_{n}$. In fact, we have the following: 
(i) $F_{n}^{k}$ has the largest number of nonzeros among all $n \times n$ ASMs $A$ such that there exist ASMs $A_{1}, \ldots, A_{k-1}, A_{k+1}, \ldots, A_{n}$ for which $\left[A_{1}, \ldots, A_{k-1}, A, A_{k+1}, \ldots, A_{n}\right]$ is an $A S H M$.

(ii) More generally, the number of nonzeros in a row (resp., column) of such an $A$ is at most equal to the number of nonzeros in the corresponding row (resp., column) of $F_{n}^{k}$.

Proof. Let $A=\left[A_{1}, A_{2}, \ldots, A_{n}\right]$ be an $n \times n \times n$ ASHM. Then row $i$ of the ASM $A_{k}$ is also row $k$ of the $i$ th row-vertical ASM plane of $A$. Hence the number of nonzeros in row $i$ of $A_{k}$ cannot exceed the minimum of the numbers of nonzeros possible in row $i$ and row $k$ of $n \times n$ ASMs, that is, cannot exceed $2 \min \{i, k\}-1$. But this is the number of nonzeros in row $i$ of the $k$ th horizontal ASM plane of the diamond ASHM $\mathfrak{D}_{n}$. Since this is true for all $i$ and $k$, the conclusions (i) and (ii) in the theorem now follow.

We remark that this proof uses strongly that we have an ASHM, not just a PASHM.

\section{$3 \quad$ ASHM Latin-like squares}

In this section we investigate ASHL's and PASHL's.

Example 6. The PASHM in (2) gives the PASHL

$$
\left[\begin{array}{llll}
1 & 3 & 2 & 4 \\
2 & 1 & 5 & 2 \\
4 & 2 & 1 & 3 \\
3 & 4 & 2 & 1
\end{array}\right]
$$

Consider the $4 \times 4 \times 4$ diamond ASHM $\mathfrak{D}_{4}=\left[F_{4}^{1}, F_{4}^{2}, F_{4}^{3}, F_{4}^{4}\right]$ given by

$$
\left[\begin{array}{llll}
1 & 0 & 0 & 0 \\
0 & 1 & 0 & 0 \\
0 & 0 & 1 & 0 \\
0 & 0 & 0 & 1
\end{array}\right] \nearrow\left[\begin{array}{rrrr}
0 & 1 & 0 & 0 \\
1 & -1 & 1 & 0 \\
0 & 1 & -1 & 1 \\
0 & 0 & 1 & 0
\end{array}\right] \nearrow\left[\begin{array}{rrrr}
0 & 0 & 1 & 0 \\
0 & 1 & -1 & 1 \\
1 & -1 & 1 & 0 \\
0 & 1 & 0 & 0
\end{array}\right] \nearrow\left[\begin{array}{llll}
0 & 0 & 0 & 1 \\
0 & 0 & 1 & 0 \\
0 & 1 & 0 & 0 \\
1 & 0 & 0 & 0
\end{array}\right] .
$$

Then the corresponding ASHL is

$$
L\left(\mathfrak{D}_{4}\right)=\left[\begin{array}{llll}
1 & 2 & 3 & 4 \\
2 & 2 & 3 & 3 \\
3 & 3 & 2 & 2 \\
4 & 3 & 2 & 1
\end{array}\right]
$$


Note that the hypermatrix in this example has the property that viewed in each of the three directions the same ASHM results and thus the same ASHL results. This is true for all diamond ASHMs.

Lemma 7. Let $A=\left[a_{i j k}\right]=\left[A_{1}, A_{2}, \ldots, A_{n}\right]$ be an $n \times n \times n$ PASHM with corresponding $n \times n$ PASHL $L(A)=1 A_{1}+2 A_{2}+\cdots+n A_{n}=\left[l_{i j}\right]$. Then the sum of the entries in each row and column of $L(A)$ equals $\left(\begin{array}{c}n+1 \\ 2\end{array}\right)$. If $A$ is an ASHM then, in addition, the following hold:

(i) The set of entries of $L(A)$ is $\{1,2, \ldots, n\}$, and the first and last rows and columns are permutations of $1,2, \ldots, n$.

(ii) Let $i, j \leq n$, and let $a_{i j k}= \pm 1$ for $k \in\left\{k_{1}, k_{2}, \ldots, k_{p}\right\}$, where $1 \leq p \leq n$ and $1 \leq k_{1}<k_{2}<\cdots<k_{p} \leq n$ and where $a_{i j k}=0$, otherwise.

(iia) If $l_{i j}=r$, then $k_{1} \leq r$, and $\left|\left\{k: a_{i j k} \neq 0\right\}\right| \leq 2 r-1$; moreover, $k_{1}=r$ implies that $p=1$. In particular, if $l_{i j}=1$, then $a_{i j 1}=1$ and $a_{i j k}=0$ for $1<k \leq n$.

(iib) If $l_{i j}=r$, then $\left|\left\{k: a_{i j k} \neq 0\right\}\right| \leq 2(n-r)+1$. In particular, if $l_{i j}=n$ then $a_{i j n}=1$ and $a_{i j k}=0$ for $1 \leq k<n$.

Proof. Consider some row $i$ of $L(A)$ (similar arguments work for a column). Since $A_{1}, A_{2}, \ldots, A_{n}$ are ASMs, row $i$ of each $A_{i}$ sums to 1 . Thus the sum of the entries of $L(A)$ in row $i$ equals

$$
\sum_{k=1}^{n} k\left(\sum_{j=1}^{n} a_{i j k}\right)=\sum_{k=1}^{n} k \cdot 1=\left(\begin{array}{c}
n+1 \\
2
\end{array}\right) .
$$

Now assume that $A$ is an ASHM. As in (ii), let $i, j \leq n$, and let $a_{i j k}=$ \pm 1 for $k \in\left\{k_{1}, k_{2}, \ldots, k_{p}\right\}$, where $1 \leq p \leq n$ and $1 \leq k_{1}<k_{2}<\cdots<$ $k_{p} \leq n$ and where $a_{i j k}=0$, otherwise. Then $p$ is odd and $a_{i j k}=1$ for $k=k_{1}, k_{3}, \ldots, k_{p}$ and $a_{i j k}=-1$ for $k=k_{2}, k_{4}, \ldots, k_{p-1}$. Thus

$l_{i j}=k_{1}+\left(-k_{2}+k_{3}\right)+\left(-k_{4}+k_{5}\right)+\cdots+\left(-k_{p-1}+k_{p}\right) \geq k_{1}+\frac{p-1}{2} \geq k_{1} \geq 1$,

and also

$$
l_{i j}=\left(k_{1}-k_{2}\right)+\left(k_{3}-k_{4}\right)+\cdots+\left(k_{p-2}-k_{p-1}\right)+k_{p} \leq k_{p}-\frac{p-1}{2} \leq k_{p} \leq n .
$$


Thus $1 \leq l_{i j} \leq n$ for each $i, j$. That the first and last rows and columns are permutations of $1,2, \ldots, n$ follows from the definitions of an ASHM and ASHL. This proves (i). Assertions (iia) and (iib) follow easily from equations (4) and (5).

The first example in Example 6 shows that assertion (iia) in Lemma 7 does not hold in general for PASHL's.

By Lemma 7, and as is the case for $n \times n$ LS's, the entries of an $n \times n$ ASHL are $\{1,2, \ldots, n\}$ but, unlike for LS's, repeats in a row or column are possible. As with LS's, we can regard an $n \times n$ ASHL $L$ as an $n \times n \times n$ (0,1)-hypermatrix

$$
H_{L}: Q_{1} \nearrow Q_{2} \nearrow \cdots \nearrow Q_{n}
$$

where an entry $k$ in the $(i, j)$-position of $L$ becomes a 1 in the $(i, j, k)$-position of $H_{L}$ giving $n \times n(0,1)$-matrices $Q_{1}, Q_{2}, \ldots, Q_{n}$. Since the entries of $H_{L}$ are $\{1,2, \ldots, n\}$, it follows that

$$
Q_{1}+Q_{2}+\cdots+Q_{n}=J_{n}
$$

a decomposition of $J_{n}$ into $n \times n(0,1)$-matrices. We call $(7)$ (and (6)) the $(0,1)$-decomposition of the ASHL $L$.

In summary, with the above notation, for the $n \times n \times n \operatorname{ASHM} A=$ $\left[A_{1}, A_{2}, \ldots, A_{n}\right]$ with corresponding Latin-like square specified by $L=\left[Q_{1}, Q_{2}, \ldots, Q_{n}\right]$, we have the two decompositions of $J_{n}$ given by

$$
J_{n}=Q_{1}+Q_{2}+\cdots+Q_{n}=A_{1}+A_{2}+\cdots+A_{n} .
$$

Example 8. The $4 \times 4$ ASHL $L$ in Example 6 gives

$H_{L}=\left[\begin{array}{llll}1 & 0 & 0 & 0 \\ 0 & 0 & 0 & 0 \\ 0 & 0 & 0 & 0 \\ 0 & 0 & 0 & 1\end{array}\right] \nearrow\left[\begin{array}{llll}0 & 1 & 0 & 0 \\ 1 & 1 & 0 & 0 \\ 0 & 0 & 1 & 1 \\ 0 & 0 & 1 & 0\end{array}\right] \nearrow\left[\begin{array}{llll}0 & 0 & 1 & 0 \\ 0 & 0 & 1 & 1 \\ 1 & 1 & 0 & 0 \\ 0 & 1 & 0 & 0\end{array}\right] \nearrow\left[\begin{array}{llll}0 & 0 & 0 & 1 \\ 0 & 0 & 0 & 0 \\ 0 & 0 & 0 & 0 \\ 1 & 0 & 0 & 0\end{array}\right]$

The next theorem asserts that an ASHL which is an ordinary LS can only arise in the classical way.

Theorem 9. Let $A=\left[a_{i j k}\right]$ be an $n \times n \times n$ ASHM. Suppose the ASHL L $(A)$ is a Latin square. Then $A$ is a permutation hypermatrix. 
Proof. Let $A=\left[A_{1}, A_{2}, \ldots, A_{n}\right]$. Since $L(A)=\left[l_{i j}\right]$ is a Latin square, it follows that for each $r=1,2, \ldots, n$, the positions of the $n r$ 's in $L(A)$ are those occupied by 1 's in an $n \times n$ permutation matrix $P_{r}$. From equation (4) in the proof of Lemma 7 we conclude that the number of nonzeros in each column $C_{i j}$ of positions $\{(i, j, k): 1 \leq k \leq n\}$ with $l_{i j}=1$ equals one. It also follows from (4) that $(i, j, k) \in C_{i j}$ implies that $k=1$. Thus $A_{1}$ is an $n \times n$ permutation matrix whose 1 's are in those positions occupied by 1 's in $L(A)$, and $A_{2}, \ldots, A_{n}$ have 0 's in those positions. Since in $L(A)$, the positions occupied by 2's are zeros in $A_{1}$, we can use a similar argument to show that $A_{2}$ is an $n \times n$ permutation matrix whose 1's are in those positions occupied by 2's in $L(A)$, and $A_{3}, \ldots, A_{n}$ have 0 's in those positions. Proceeding inductively, we conclude that $A$ is a permutation hypermatrix.

Recall that, for vectors $x=\left(x_{1}, x_{2}, \ldots, x_{n}\right)$ and $y=\left(y_{1}, y_{2}, \ldots, y_{n}\right), x$ is majorized by $y$, written $x \preceq y$, whenever $\sum_{j=1}^{k} x_{[j]} \leq \sum_{j=1}^{k} y_{[j]}(k \leq n)$ with equality for $k=n$. Here $x_{[j]}$ denotes the $j$ th largest component in $x$. We now define a new order for matrices based on majorization. Let $A$ and $B$ be $m \times n$ matrices. We say that $A$ is line majorized by $B$ if each line (row or column) of $A$ is majorized by the corresponding line in $B$, and then we write $A \preceq_{l} B$. This is a preorder on the class of $m \times n$ matrices. Let $\operatorname{Row}_{i}(A)$ denote the $i$ th row of a matrix $A$.

Theorem 10. Let $A$ be an $n \times n \times n A S H M$ and $P$ an $n \times n \times n$ permutation ASHM. Then

$$
L(A) \preceq_{l} L(P),
$$

that is, each row of the ASHL $L(A)$ is majorized by $z_{n}=(n, n-1, \ldots, 1)$.

Proof. First note that each row of $L(P)$ is a permutation of $z_{n}=(n, n-$ $1, \ldots, 1)$ (recall, $n$-tuples are identified with column vectors). Let $i \leq n$. Let $C$ be the $i$ th column-vertical plane of $A$ so that $C$ is an ASM. The $i$ th row in $L(A)$ satisfies

$$
\operatorname{Row}_{i}(L(A))=z_{n}^{T} C .
$$

(where we view the matrix $C$ so the layers of $A$ are organized downwards). Let $B=C^{T}$, so $B$ is also an ASM, and define $w=B z_{n}=\left(z_{n}^{T} C\right)^{T}$. We need to show the majorization

$$
(*) \quad w \preceq z_{n} .
$$


Let $e^{(k)}=e_{1}+e_{2}+\cdots+e_{k}(k \leq n)$ where $e_{j}$ is the $j$ th unit vector. So

$$
z_{n}=\sum_{k=1}^{n} e^{(k)} .
$$

Then

$$
w=B z_{n}=B \sum_{k=1}^{n} e^{(k)}=\sum_{k=1}^{n} B e^{(k)}=\sum_{k=1}^{n} v^{(k)}
$$

where $v^{(k)}=B e^{(k)}(k \leq n)$. Then $v^{(k)}$ is the sum of the first $k$ columns of $B$, so $v^{(k)}$ is a $(0,1)$-vector, as $B$ is an ASM. Moreover, $v^{(k)}$ has $k$ ones. Let $V$ be the $n \times n$ matrix whose columns are $v^{(1)}, v^{(2)}, \ldots, v^{(n)}$. Then $V$ is a $(0,1)$ matrix with column sum vector $(1,2, \ldots, n)$ and row sum vector $w$, by (9). We may therefore apply the (simple part of the) Gale-Ryser theorem, and conclude that $w$ is majorized by the conjugate $(1,2, \ldots, n)^{*}$ of the column sum vector $(1,2, \ldots, n)$. But $(1,2, \ldots, n)^{*}=z_{n}$, so we have shown that $w \preceq z_{n}$, and hence the majorization in $(*)$ holds. Similarly, one shows the majorization for columns of $L(A)$ and $L(P)$.

Consider again the diamond ASHM $\mathfrak{D}_{n}$. The matrix $L\left(\mathfrak{D}_{n}\right)$ remains the same if its rows and also its columns are simultaneously reversed. Moreover, when $i \leq\lfloor n / 2\rfloor$, then its $i$ th row is

$\operatorname{Row}_{i}\left(L\left(\mathfrak{D}_{n}\right)\right)=v^{(i)}:=(i, i, \ldots, i, i+1, i+2, \ldots, n-i+1, n-i+1, \ldots, n-i+1)$

where the first (resp. last) $i$ entries are equal.

Example 11. Let $n=7$. Then

$$
L\left(\mathfrak{D}_{7}\right)=\left[\begin{array}{lllllll}
1 & 2 & 3 & 4 & 5 & 6 & 7 \\
2 & 2 & 3 & 4 & 5 & 6 & 6 \\
3 & 3 & 3 & 4 & 5 & 5 & 5 \\
4 & 4 & 4 & 4 & 4 & 4 & 4 \\
5 & 5 & 5 & 4 & 3 & 3 & 3 \\
6 & 6 & 5 & 4 & 3 & 2 & 2 \\
7 & 6 & 5 & 4 & 3 & 2 & 1
\end{array}\right] .
$$

Consider an ASHM $A$, whose first three horizontal planes coincide with those of $\mathfrak{D}_{7}$ while the remaining planes are suitable permutation matrices 
$P_{4}, P_{5}, P_{6}, P_{7}$. The sum of these three horizontal planes is

$$
S=\left[\begin{array}{lllllll}
1 & 1 & 1 & 0 & 0 & 0 & 0 \\
1 & 1 & 0 & 1 & 0 & 0 & 0 \\
1 & 0 & 1 & 0 & 1 & 0 & 0 \\
0 & 1 & 0 & 1 & 0 & 1 & 0 \\
0 & 0 & 1 & 0 & 1 & 0 & 1 \\
0 & 0 & 0 & 1 & 0 & 1 & 1 \\
0 & 0 & 0 & 0 & 1 & 1 & 1
\end{array}\right] .
$$

Then $P_{4}+P_{5}+P_{6}+P_{7}=J_{7}-S$. By specifically choosing $P_{4}$ to be the permutation matrix

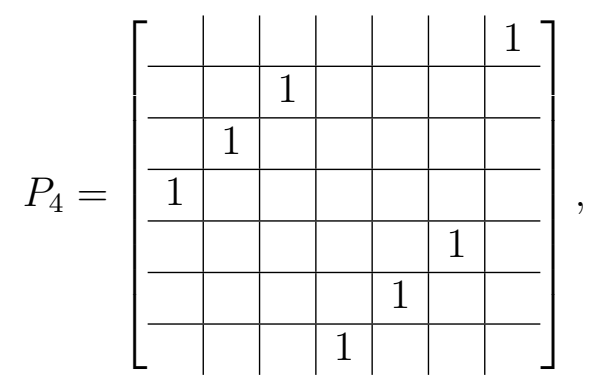

we can obtain

$$
L(A)=\left[\begin{array}{lllllll}
1 & 2 & 3 & 7 & 5 & 6 & 4 \\
2 & 2 & 3 & 3 & 6 & 7 & 5 \\
3 & 3 & 2 & 5 & 3 & 5 & 7 \\
4 & 3 & 4 & 2 & 6 & 3 & 6 \\
6 & 7 & 3 & 4 & 2 & 3 & 3 \\
7 & 5 & 6 & 3 & 3 & 2 & 2 \\
5 & 6 & 7 & 4 & 3 & 2 & 1
\end{array}\right]
$$

For instance, concerning row four in $L(A)$ we have the majorization

$$
(4,3,4,2,6,3,6) \preceq z_{7}=(7,6,5,4,3,2,1)
$$

which is in accordance with Theorem 10. In this example, we can also check that every row or column of $L(A)$ majorizes the corresponding row or column of $L\left(\mathfrak{D}_{7}\right)$, so $L\left(\mathfrak{D}_{7}\right) \preceq_{l} L(A)$.

In an $n \times n$ LS, each integer in $\{1,2, \ldots, n\}$ occurs exactly $n$ times. In an $n \times n$ ASHL, the entries are also taken from $\{1,2, \ldots, n\}$, but their 
multiplicity can vary. In the $7 \times 7$ ASHL in (10), the integer 3 occurs 14 times. This example can be generalized to all odd $n \geq 5$ giving an $n \times n$ ASHL in which $(n-1) / 2$ occurs $2 n$ times.

In view of Example 11 one may ask if $L\left(\mathfrak{D}_{n}\right) \preceq_{l} L(A)$ holds for every ASHM $A$ of size $n \times n \times n$. As shown by the next example, this is not the case.

Example 12. Consider the ASHM $A^{\prime}$ given by
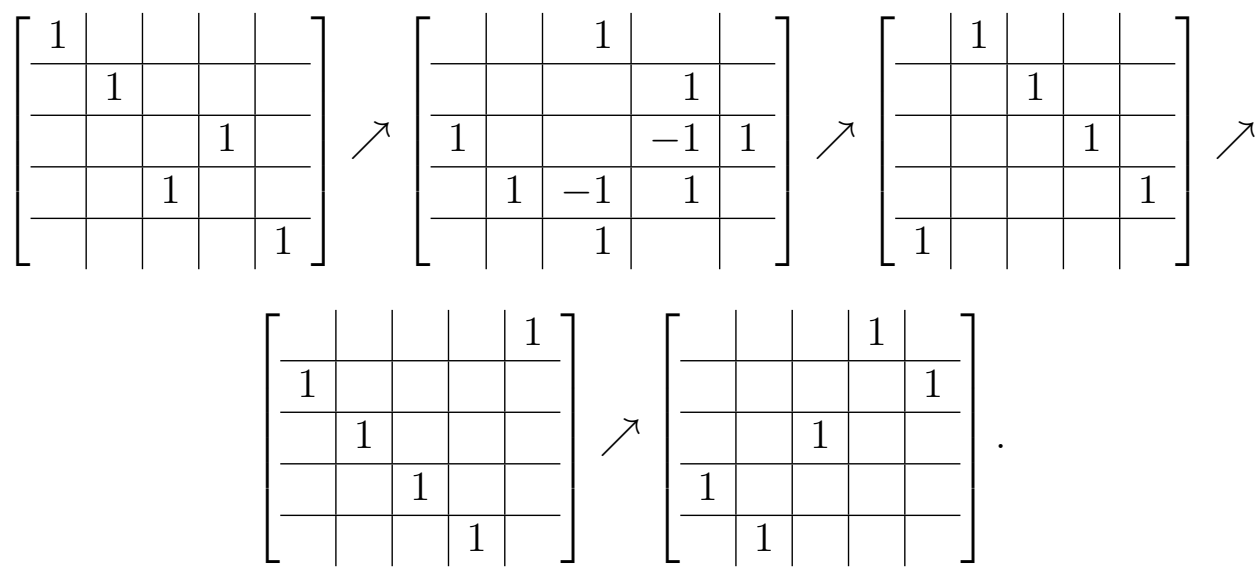

Let $A$ be the ASHM obtained from $A^{\prime}$ by letting the horizontal planes of $A^{\prime}$ become the column-vertical planes of $A$. Let $x$ denote the second row of the ASHL $L(A)$ associated with $A$. Then $x$ is computed from the second matrix above (the only one with some negative entries) and we get

$$
x=(3,2,4,3,3) .
$$

Consider the diamond $\mathfrak{D}_{5}$. Then (see above) the second row of $L\left(\mathfrak{D}_{5}\right)$ is $(2,2,3,4,4)$. But $(2,2,3,4,4) \npreceq(3,2,4,3,3)$ because $4+4>4+3$.

We say that a line in a matrix is constant if all its entries are equal. Let $A=\left[A_{1}, A_{2}, \ldots, A_{n}\right]$ be an $n \times n \times n$ ASHM and let $L=L(A)$ be its corresponding ASHL. Assume that $L$ has a constant line. Then $n$ must be odd. In fact, by Lemma 7, the sum of the entries in any line of $L$ is $n(n+1) / 2$, so in the line which is constant each entry must be equal to $(n+1) / 2$. But every entry in $L$ is integral, so $n$ must be odd.

For instance, for the diamond ASHM $\mathfrak{D}_{n}$, where $n$ is odd, say $n=2 k-1$, the $k$ 'th row and column of the ASHL $L\left(\mathfrak{D}_{n}\right)$ are constant lines. The case of $n=7$ is shown in Example 11. 
Example 13. Consider the ASM

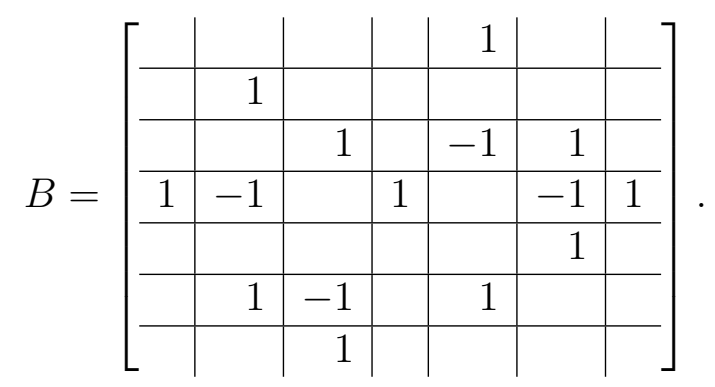

Then $z_{7}^{T} B=(4,4,4,4,4,4,4)$. It is possible to construct an ASHM $A$ such that $B$ is the third column-vertical plane; this follows from results we establish in the next section. Thus, the third row of the corresponding ASHL $L(A)$ is $(4,4, \ldots, 4)$. Note that this vector is minimal in the majorization order in $\mathbb{R}^{7}$. On the other hand, the first row of $L(A)$ is a permutation of $(1,2, \ldots, 7)$ which, by Theorem 10 , is maximal in the majorization order among all possible rows of an ASHL of order 7 .

The ASHL of a permutation hypermatrix does not contain any constant line, except in the trivial case of $n=1$. For $n=3$, the ASHL $L\left(\mathfrak{D}_{3}\right)$ has a constant line, both a row and a column. An ASHM where every line contains at most one -1 will be called a near-permutation hypermatrix. We now show that near-permutation ASHMs have this same property as permutation ASHMs, namely the corresponding ASHL's do not have any constant line (when $n>3$ ).

Theorem 14. Let $A=\left[A_{1}, A_{2}, \ldots, A_{n}\right]$ be an $n \times n \times n$ near-permutation $A S H M$ where $n>3$. Then the ASHL $L(A)$ does not have any constant lines.

Proof. Assume $L=L(A)$ has a constant line, say row $i$. As explained above, $n$ must be odd, so $n=2 k-1$ with $k \geq 3$, and every entry in row $i$ of $L$ must be $(n+1) / 2=k$. Let $B=\left[b_{i j}\right]$ be the column-vertical plane of $A$ associated with row $i$ of $L$; this row equals $z_{n}^{T} B$ and all entries are $k$. Then $B$ cannot be a permutation matrix, because then row $i$ of $L$ is a permutation of $(1,2, \ldots, n)$. So, some line in $B$ has a negative entry. On the other hand, no line in $B$ can have more than one negative entry, as $A$ is a near-permutation ASHM. The first column of $B$ is a unit vector, so its 1 must be in row $k$, i.e., $b_{k 1}=1$. This implies that the second column cannot be a unit vector, because then its 1 would be in another row than the $k$ th, and then $z$ times 
that column would be different from $k$. So, the second column of $B$ contains a -1 , which then must be in row $k$, again by the alternating property of $B$. By similar arguments, $b_{k n}=1$ and $b_{k, n-1}=-1$. However, as $n>3$, this means that row $k$ in $B$ has two -1 's; a contradiction. This proves that no row in $L$ is constant, and similar arguments show that no column is constant.

For ASHMs with more than one negative entry in some lines, the situation is more complicated. However, the construction in the previous proof gives the following property.

Corollary 15. Let $A=\left[A_{1}, A_{2}, \ldots, A_{n}\right]$ be an $n \times n \times n$ ASHM with corresponding $A S H L L(A)$. If $n>3$, then the second (resp. second-last) row or column in $L(A)$ is not constant.

Proof. We can use the same arguments as in the proof of Theorem 14. The only change is that the ASM $B$ cannot have more than one negative entry in any line, because this would contradict that $B$ is the second column-vertical plane of $A$ (as, in that case, the first column-vertical plane has two ones in the same line).

Example 13 shows that the third row or column of an ASHL may be constant.

Let again $z_{n}=(n, n-1, \ldots, 2,1)$. The rows and columns of an $n \times n$ ASHL are obtained by vector-matrix multiplications

$$
z_{n}^{T} A=v(A)=\left(v_{1}, v_{2}, \ldots, v_{n}\right) \text { and } A^{T} z_{n}=h(A)=\left(h_{1}, h_{2}, \ldots, h_{n}\right)
$$

where $A$ is some $n \times n$ ASM. We call $v(A)$ in (11) the weighted vertical projection of the ASM $A$, and $h(A)$ the weighted horizontal projection. It follows from Theorem 10 that the weighted projections of an $n \times n$ ASM are majorized by $z_{n}$. Thus the weighted projections of an ASM can be regarded as "integral smoothings" of the entries of $z_{n}$. As we know, the weighted vertical and horizontal projections of the diamond ASMs are the $n$-vectors $\left(\frac{n+1}{2}, \frac{n+1}{2}, \ldots, \frac{n+1}{2}\right)$ if $n$ is odd and $\left(\frac{n}{2}+1, \ldots, \frac{n}{2}+1, \frac{n}{2}, \ldots, \frac{n}{2}\right)$, with $n / 2$ each of the two different components, if $n$ is even. If $A$ is a permutation matrix $P$ corresponding to the permutation $\left(i_{1}, i_{2}, \ldots, i_{n}\right)$ of $\{1,2, \ldots, n\}$, then $h(P)=\left(i_{1}, i_{2}, \ldots, i_{n}\right)$, and $v(P)=\left(j_{1}, j_{2}, \ldots, j_{n}\right)$ where $\left(j_{1}, j_{2}, \ldots, j_{n}\right)$ is the inverse permutation. So either of $v(P)$ and $h(P)$ determines $P$. This is in contrast to ASMs in general where two different ASMs can have both the same weighted horizontal projections and the same weighted vertical projections. 
Example 16. Let

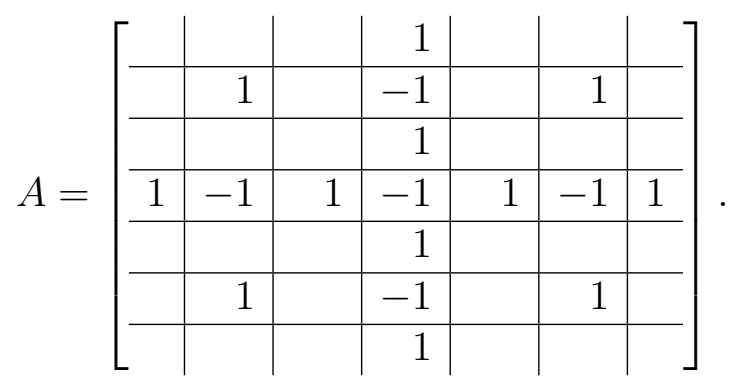

Then $A \neq D_{7}$ but $v(A)=h(A)=(4,4,4,4,4,4,4)=h\left(D_{7}\right)=v\left(D_{7}\right)$.

In the next lemma, we show that if the weighted projection of an $n \times n$ ASM $A$ is a permutation of $\{1,2, \ldots, n\}$, then $A$ is a permutation matrix. Thus, if $A$ is not a permutation matrix, then $z_{n}^{T} A$ must have a repeated entry.

Lemma 17. Let $A=\left[a_{i j}\right]$ be an $n \times n$ ASM. If $z_{n}^{T} A=z_{n}^{T} P$ for some $n \times n$ permutation matrix $P$, then $A=P$.

Proof. Let $v=\left(i_{1}, i_{2}, \ldots, i_{n}\right)=z_{n}^{T} P=z_{n} A$, where $v$ is a permutation of $\{1,2, \ldots, n\}$. Let $k$ be such that $i_{k}=1$. Since the +1 's and -1 's of $A$ alternate in the columns beginning and ending with a +1 , it follows that column $k$ of $A$ must equal $(0,0, \ldots, 0,1)$; since $A$ is an ASM, the last row of $A$ has only zeros in columns different from $k$. Thus, the last row and column $k$ of $A$ agree with the corresponding row and column of $P$.

Now let $l$ be such that $i_{l}=2$. Since column $l$ of $A$ has a zero in row $n$, column $l$ of $A$ cannot contain three or more nonzeros, for if it did, $u$ times column $l$ of $A$ would be more than 2. It follows that column $l$ of $A$ equals $(0,0, \ldots, 0,1,0)$, and the row $n-1$ of $A$ has only zeros in columns different from $l$. Hence $A$ agrees with $P$ in both row $n-1$ and column $l$. Continuing like this, if follows by induction that $A=P$.

Corollary 18. Let $A$ be an $n \times n \times n$ ASHM with corresponding $A S H L L(A)$. If $n-1$ rows (resp., columns) of $L(A)$ are permutations of $\{1,2, \ldots, n\}$, then $A$ is an $n \times n \times n$ permutation hypermatrix and $L(A)$ is a Latin square.

Proof. By Lemma 17, $n-1$ of the column-vertical planes of $A$ are permutation matrices. Since $A$ is an ASHM, it follows that the remaining columnvertical plane is also a permutation matrix; hence $A$ is a permutation hypermatrix and so $L$ is a Latin square. 
We conclude this section with the following variation of the majorization order. For vectors $x=\left(x_{1}, x_{2}, \ldots, x_{n}\right)$ and $y=\left(y_{1}, y_{2}, \ldots, y_{n}\right)$ (not assumed to be monotone), we write $x \preceq^{*} y$ provided that

$$
\sum_{j=1}^{p} x_{j} \leq \sum_{j=1}^{p} y_{j} \quad(p \leq n)
$$

where equality holds for $p=n$. Note that we only require inequalities to hold for the leading partial sums. For an $n \times n$ matrix $A$, let $h(A)$ denote its weighted horizontal projection, i.e., $h(A)=A^{T} z_{n}$ where $z_{n}=(n, n-1, \ldots, 1)$.

Theorem 19. Let $A$ be an $n \times n$ ASM. Let $p=\sigma_{-}(A)$. Then there is a sequence of $n \times n$ ASMs

$$
A^{(p)}, A^{(p-1)}, \ldots, A^{(0)}
$$

where $\left(\right.$ i) $A^{(p)}=A$, (ii) $A^{(0)}$ is a permutation matrix, (iii) $\sigma_{-}\left(A^{(s)}\right)=s$ $(s=0,1, \ldots, p)$, and (iv) the following majorizations hold

$$
h\left(A^{(p)}\right) \preceq^{*} h\left(A^{(p-1)}\right) \preceq^{*} \cdots \preceq^{*} h\left(A^{(0)}\right) .
$$

Proof. If $p=0$ ( $A$ is a permutation matrix), there is nothing to prove, so assume $p=\sigma_{-}(A) \geq 1$ where $A=\left[a_{i j}\right]$ is an ASM. Choose $(k, l)$ with $k+l$ minimal such that $a_{k l}=-1$. Then there is a unique $l^{\prime}<l$ with $a_{k l^{\prime}}$ nonzero, and we have $a_{k l^{\prime}}=1$. Similarly, there is a unique $k^{\prime}<k$ with $a_{k^{\prime} l}$ nonzero and $a_{k^{\prime} l}=1$. By the choice of $k, l$, there is no negative entry in the leading $k \times l$ submatrix, apart from $a_{k l}$. Moreover, $a_{k^{\prime} l^{\prime}}=0$. Let $B=\left[b_{i j}\right]$ be obtained from $A$ by letting $b_{k l}=b_{k^{\prime} l}=b_{k l^{\prime}}=0, b_{k^{\prime} l^{\prime}}=1$, and, otherwise, $b_{i j}=a_{i j}$. Then $B$ is an ASM and $\sigma_{-}(B)=\sigma_{-}(A)-1$.

Moreover, $B$ is obtained from $A$ by adding the submatrix

$$
K=\left[\begin{array}{rr}
1 & -1 \\
-1 & 1
\end{array}\right]
$$

to the submatrix of $A$ corresponding to rows $k^{\prime}<k$ and columns $l^{\prime}<l$. Thus, denoting the $s^{\prime}$ th component of $h(A)$ and $h(B)$ by $h(A)_{s}$ and $h(B)_{s}$, respectively,

$$
\begin{aligned}
& h(B)_{l^{\prime}}=h(A)_{l^{\prime}}+\left(k-k^{\prime}\right), \quad h(B)_{l}=h(A)_{l}-\left(k-k^{\prime}\right), \text { and } \\
& h(B)_{i}=h(A)_{i} \text { for all other } i .
\end{aligned}
$$


This implies that

$$
h(B) \preceq^{*} h(A) .
$$

We now repeat this process with $A$ replaced by $B$. Clearly, after $p$ such operations, we have produced a sequence of ASMs with all the desired properties.

Example 20. Consider the following ASM $A$ with $h(A)=(2,3,3,4,3)$ :

$$
A=A^{(2)}=\left[\begin{array}{l|l|l|l|l} 
& & & + & \\
\hline & + & & & \\
\hline & & + & - & + \\
\hline+ & - & & + & \\
\hline & + & & &
\end{array}\right] .
$$

Adding the matrix $K$ to the submatrix induced by rows 2,4 and columns 1,2 gives

$$
A^{(1)}=\left[\begin{array}{l|l|l|l|l} 
& & & + & \\
\hline+ & & & & \\
\hline & & + & - & + \\
\hline & & & + & \\
\hline & + & & &
\end{array}\right]
$$

with $w\left(A^{(1)}\right)=(4,1,3,4,3)$. Finally, adding $K$ to the submatrix induced by rows 1,3 and columns 3,4 gives the permutation matrix

$$
A^{(0)}=\left[\begin{array}{l|l|l|l|l} 
& & + & & \\
\hline+ & & & & \\
\hline & & & & + \\
\hline & & & + & \\
\hline & + & & &
\end{array}\right]
$$

with $w\left(A^{(0)}\right)=(4,1,5,2,3)$. Here we have the majorizations

$$
(2,3,3,4,3) \preceq^{*}(4,1,3,4,3) \preceq^{*}(4,1,5,2,3) .
$$




\section{Completion problems}

In this section we study completion problems where some ASMs are given as horizontal planes and one wants to extend them to obtain an ASHM.

Consider a $(0, \pm 1)$-vector $x=\left(x_{1}, x_{2}, \ldots, x_{p}\right)$. We say that $x$ is $(1, *)$ alternating if its nonzeros (if any) alternate in sign and the first nonzero is a 1 . Similarly, $x$ is $(*, 1)$-alternating if its nonzeros (if any) alternate in sign and the last nonzero is a 1 . Thus all the lines of an ASM are both $(1, *)$-alternating and $(*, 1)$-alternating.

The $i$ th row (resp. column) of a matrix $X$ is denoted by $\operatorname{row}_{i}(X)$ (resp. $\left.\operatorname{col}_{i}(X)\right)$. As before the matrix $J_{n}$ is the all ones matrix of order $n$.

Let $(n-1) n \times n$ ASMs $A_{1}, \ldots, A_{k-1}, A_{k+1}, \ldots, A_{n}$ be given, and let $A_{(k)}$ denote the corresponding $n \times n \times(n-1)$ hypermatrix. We consider the question: When does there exist an $n \times n \mathrm{ASM} A_{k}$ such that $A=$ $\left[A_{1}, \ldots, A_{k-1}, A_{k}, A_{k+1}, \ldots, A_{n}\right]$ is an ASHM? If the answer is affirmative, we say that $A_{(k)}$ has an $A S H M$-completion $A$ at (horizontal) layer $k$. Let $A_{(k)}=\left[a_{i j s}\right]$, where $s \in\{1,2, \ldots, n\} \backslash\{k\}$. An obvious necessary condition for $A_{(k)}$ to have an ASHM-completion at layer $k$ is

(i) $\left(a_{i j 1}, \ldots, a_{i j, k-1}\right)$ is $(1, *)$-alternating $(1 \leq i, j \leq n)$,

(ii) $\left(a_{i j, k+1}, \ldots, a_{i j n}\right)$ is $(*, 1)$-alternating $(1 \leq i, j \leq n)$.

For the given $A_{(k)}$, (12) implies that $\sum_{s \neq k} A_{s}$ is a $(0,1,2)$-matrix.

Theorem 21. Let $A_{1}, \ldots, A_{k-1}, A_{k+1}, \ldots, A_{n}$ be $(n-1) n \times n$ ASMs. Let $A_{(k)}$ be the corresponding $n \times n \times(n-1)$ hypermatrix and let $X^{(k)}=\sum_{s \neq k} A_{s}$. Define $Y=J_{n}-X^{(k)}$. Then $A_{(k)}$ has an ASHM-completion at layer $k$ if and only if both (12) and the following majorization conditions hold

$$
\begin{array}{rlr}
\text { (i) } \quad \operatorname{row}_{i}\left(X^{(k)}\right) & \preceq^{*}(1,1, \ldots, 1,0) & (i \leq n), \\
\text { (ii) } \quad \operatorname{col}_{j}\left(X^{(k)}\right) & \preceq^{*}(1,1, \ldots, 1,0) & (j \leq n) .
\end{array}
$$

If these conditions hold, there is a unique ASHM-completion at layer $k$ which is $A=\left[A_{1}, \ldots, A_{k-1}, Y, A_{k+1}, \ldots, A_{n}\right]$.

Proof. Let $X^{(k)}=\left[x_{i j}^{(k)}\right]$. Assume first that $A=\left[A_{1}, \ldots, A_{k-1}, Z, A_{k+1}, \ldots, A_{n}\right]$ is an ASHM-completion of layer $k$ of $A_{(k)}$. Then every line sum of $A$ is 1 , so the sum of all these matrices (the $n$ layers) is $J_{n}$, and therefore $Z=$ $J_{n}-X^{(k)}=Y$. Thus, such a completion is unique, if it exists. Also, condition (12) holds as every line is alternating. Since $Z=Y=\left[y_{i j}\right]$ is an ASM, 
for every $p<n$, the sum of its first $p$ entries in a row is nonnegative, so for each $i \leq n$,

$$
\text { (*) } 0 \leq \sum_{j=1}^{p} y_{i j}=\sum_{j=1}^{p}\left(1-x_{i j}^{(k)}\right)=p-\sum_{j=1}^{p} x_{i j}^{(k)},
$$

that is, $\sum_{j=1}^{p} x_{i j}^{(k)} \leq p$. Moreover,

$$
\sum_{j=1}^{n} x_{i j}^{(k)}=\sum_{j=1}^{n} \sum_{s \neq k} a_{i j s}=\sum_{s \neq k} \sum_{j=1}^{n} a_{i j s}=(n-1) \cdot 1=n-1 .
$$

This proves that $\operatorname{row}_{i}\left(X^{(k)}\right) \preceq^{*}(1,1, \ldots, 1,0)$ for each $i \leq n$. Similarly, one obtains $\operatorname{col}_{j}\left(X^{(k)}\right) \preceq^{*}(1,1, \ldots, 1,0)$ for each $j \leq n$. This shows the necessity of the condition.

Conversely, assume that conditions (12) and (13) hold. Define

$$
A=\left[A_{1}, \ldots, A_{k-1}, Y, A_{k+1}, \ldots, A_{n}\right]=\left[a_{i j s}\right] .
$$

We verify that $A$ is an ASHM. Since $Y=J_{n}-X^{(k)}$ and $X^{(k)}=\sum_{s \neq k} A_{s}$, $\sum_{s} a_{i j s}=1$ for each $i, j \leq n$. Let $i, j \leq n$. Consider the line $\left(a_{i j 1}, a_{i j 2}, \ldots, a_{i j n}\right)$ in $A$, and its subvectors $u=\left(a_{i j 1}, a_{i j 2}, \ldots, a_{i j, k-1}\right)$ and $v=\left(a_{i j, k+1}, a_{i j, k+2}, \ldots, a_{i j n}\right)$. By $(12) u$ is $(1, *)$-alternating and $v$ is $(*, 1)$-alternating. Define $\sigma_{1}=\sum_{s=1}^{k-1} a_{i j s}$ and $\sigma_{2}=\sum_{s=k+1}^{n} a_{i j s}$. Then we have the following cases:

(i) $\sigma_{1}=\sigma_{2}=0$. Then the last nonzero of $u$ (if any) is -1 , and the first nonzero of $u$ (if any) is -1 . Moreover, $a_{i j k}=y_{i j}=1$, so the line is alternating. (ii) $\sigma_{1}=1, \sigma_{2}=0$. Then the last nonzero of $u$ is 1 , and the first nonzero of $v$ (if any) is -1 , and $a_{i j k}=y_{i j}=1-(0+1)=0$, so the line is alternating. (iii) $\sigma_{1}=0, \sigma_{2}=1$. Similar to case (ii).

(iv) $\sigma_{1}=1, \sigma_{2}=1$. Then the last nonzero of $u$ (if any), and the first nonzero of $v$, is 1 , and $a_{i j k}=y_{i j}=1-(1+1)=-1$, so the line is alternating.

It only remains to show that $Y$ is an ASM. Let $i \leq n$. The computation in $(*)$ shows that, for each $p<n, \sum_{j=1}^{p} y_{i j} \geq 0$ as $\sum_{j=1}^{p} x_{i j}^{(k)} \leq p$ by (12). Moreover, as each $A_{s}$ is an ASM,

$\sum_{j=1}^{n} y_{i j}=n-\sum_{j=1}^{n} x_{i j}^{(k)}=n-\sum_{j=1}^{n} \sum_{s \neq k} a_{i j s}=n-\sum_{s \neq k} \sum_{j=1}^{n} a_{i j s}=n-(n-1) \cdot 1=1$.

Similarly, we obtain, for each $j \leq n$, that $\sum_{i=1}^{p} y_{i j} \geq 0$ for each $p<n$, and $\sum_{i=1}^{n} y_{i j}=1$. This shows that $Y$ is an ASM, and therefore $A$ is an ASHM. 
In what follows we shall make use of the following classical decomposition result $([3,8])$.

Theorem 22. Let $A$ be an $n \times n$ nonnegative integral matrix with equal row and column sums, say equal to $k$. Then $A$ may be decomposed as the sum of $k$ permutation matrices.

Let $k<n$ and let $A=\left[A_{1}, A_{2}, \ldots, A_{k}\right]$ be an $n \times n \times k$ hypermatrix where $A_{1}, A_{2}, \ldots, A_{k}$ are ASMs. We consider the problem of extending $A$ by inserting $(n-k) n \times n$ ASMs $A_{k+1}, \ldots, A_{n}$ so that $A^{\prime}=\left[A_{1}, \ldots, A_{k}, A_{k+1}, \ldots, A_{n}\right]$ is an $n \times n \times n$ ASHM. An obvious necessary condition on the vertical lines is the following:

$$
\left(a_{i j 1}, \ldots, a_{i j k}\right) \text { is }(1, *) \text {-alternating }(1 \leq i, j \leq n) .
$$

The next theorem says that no other condition is needed.

Theorem 23. Let $k<n$, and let $A_{1}, A_{2}, \ldots, A_{k}$ be $k n \times n$ ASMs satisfying (14). Then there exist $n \times n$ ASMs $A_{k+1}, \ldots, A_{n}$ such that $A^{\prime}=$ $\left[A_{1}, \ldots, A_{k}, A_{k+1}, \ldots, A_{n}\right]$ is an $n \times n \times n$ ASHM. Moreover, each of these additional $A S M s A_{j}(k<j \leq n)$ may be chosen as a permutation matrix.

Proof. Let $S=\sum_{s=1}^{k} A_{s}$. Then each line sum in $S$ is $k$. Moreover, it follows from condition (14) that $S$ is a $(0,1)$-matrix. Let $T=J_{n}-S$ which is also a $(0,1)$-matrix with each line sum $n-k$. Clearly $S$ and $T$ have disjoint supports.

Note that if $S$ has a 1 in position $(i, j)$ it means that $\sum_{s=1}^{k} a_{i j s}=1$, and the vertical line $\left(a_{i j 1}, \ldots, a_{i j k}\right)$ is alternating. Since all line sums in $T$ are $n-k$, by Theorem 22 , there are permutation matrices $A_{k+1}, \ldots, A_{n}$ such that

$$
T=\sum_{s=k+1}^{n} A_{s} .
$$

Define the $n \times n \times n$ hypermatrix $A^{\prime}=\left[A_{1}, \ldots, A_{k}, A_{k+1}, \ldots, A_{n}\right]=\left[a_{i j s}\right]$. Then $A^{\prime}$ is an ASHM because:

(i) In positions $(i, j)$ where $S$ has a 1 the line $\left(a_{i j 1}, \ldots, a_{i j k}\right)$ is alternating and has sum 1 while $a_{i j s}=0$ for $s>k$.

(ii) In positions $(i, j)$ where $S$ has a $0, T$ has a 1 , so exactly one $A_{s}$, for $s>k$, has a 1 in that position $(i, j)$. Moreover, the line $\left(a_{i j 1}, \ldots, a_{i j k}\right)$ is $(1, *)$ alternating so its last nonzero, if any, is -1 . So the whole line $\left(a_{i j 1}, \ldots, a_{i j n}\right)$ is alternating. 
We remark that one may find the permutation matrices $A_{k+1}, \ldots, A_{n}$ in Theorem 23 efficiently (in polynomial time) since matching algorithms may be used to find the decomposition in Theorem 22 .

Example 24. Consider Theorem 23, and its proof, with $k=2$ and

$$
A_{1}=\left[\begin{array}{l|l|l|l} 
& 1 & & \\
\hline & & 1 & \\
\hline 1 & & & \\
\hline & & & 1
\end{array}\right], \quad A_{2}=\left[\begin{array}{l|r|r|r} 
& & 1 & \\
\hline 1 & & -1 & 1 \\
\hline & 1 & & \\
\hline & & 1 &
\end{array}\right] .
$$

Then

$$
S=\left[\begin{array}{l|l|l|l} 
& 1 & 1 & \\
\hline 1 & & & 1 \\
\hline 1 & 1 & & \\
\hline & & 1 & 1
\end{array}\right], \quad T=\left[\begin{array}{l|l|l|l}
1 & & & 1 \\
\hline & 1 & 1 & \\
\hline & & 1 & 1 \\
\hline 1 & 1 & &
\end{array}\right] .
$$

So, we can extend $\left[A_{1}, A_{2}\right]$ into an ASHM by adding the permutation matrices

$$
A_{3}=\left[\begin{array}{l|l|l|l}
1 & & & \\
\hline & & 1 & \\
\hline & & & 1 \\
\hline & 1 & &
\end{array}\right], \quad A_{4}=\left[\begin{array}{l|l|l|l} 
& & & 1 \\
\hline & 1 & & \\
\hline & & 1 & \\
\hline 1 & & &
\end{array}\right] .
$$

Recall that $\sigma_{-}(A)$ denotes the number of negative entries of a matrix, or hypermatrix, $A$. By Theorem 5 the maximum number $m_{n}$ of nonzeros in an $n \times n \times n$ ASHM is given by $m_{n}=\frac{n\left(n^{2}+2\right)}{3}$. Therefore the maximum number $m_{n}^{-}$of -1 's in an ASHM is

$$
m_{n}^{-}=\sigma_{-}\left(\mathfrak{D}_{n}\right)=m_{n}-n^{2}=\frac{n\left(n^{2}-3 n+2\right)}{3} .
$$

Let $n \geq 3$. Our goal now is to show that, for any nonnegative integer $t \leq m_{n}^{-}$, there exists an ASHM $A$ with $\sigma_{-}(A)=t$ (for $n \leq 2$ there is nothing to show).

First, we describe a class of ASMs that are obtained from the diamond ASMs. The construction is illustrated in Example 26. Let $1<k<n$ and consider $F_{n}^{k}$. For $1 \leq j \leq k$, the $j^{\prime}$ th positive diagonal of $F_{n}^{k}$ consists of the positions $(j, k-j+1),(j+1, k-j+2), \ldots,(n+j-k, n-j+1)$. Similarly, for $j \leq k-1$ the $j^{\prime}$ th negative diagonal of $F_{n}^{k}$ consists of the 
positions $(j+1, k-j+1),(j+2, k-j+2), \ldots,(n+j-k, n-j+2)$. Define $p=n-k$ which is the number of -1 's in each of the negative diagonals of $F_{n}^{k}$. We have $\sigma_{-}\left(F_{n}^{k}\right)=(k-1)(n-k)$. Let $r$ and $s$ be integers with $0 \leq r<k$ and $0 \leq s<n-k$. Let $F_{n}^{k, r, s}=\left[a_{i j}\right]$ be the $n \times n$ matrix obtained from $F_{n}^{k}$ as follows:

(i) If $r>0$, let each entry in the first $r$ positive diagonals and the first $r$ negative diagonals be zero (some of these entries may be changed again in step (ii));

(ii) If $p$ is even, let $a_{1 n}=a_{2, n-1}=\cdots=a_{r, n-r+1}=1$. Otherwise, when $p$ is odd, there are two subcases: (a) $r$ is odd; then let the entry be 1 in each of the $r$ first positions in the sequence $(1, n),(2, n-2),(3, n-1),(4, n-4)$, $(5, n-3), \ldots$; (b) $r$ is even; let the entry be 1 in each of the $r$ first positions in the sequence $(1, n-1),(2, n),(3, n-3),(4, n-2), \ldots$.

(iii) If $s>0$, let the last $s$ entries in the last negative diagonal be zero, and the last $s+1$ entries of the last positive diagonal be zero. Finally, let $a_{k+s, 1}=1$.

Define

$K_{n, k}=\{(i, i+k-1): 1 \leq i \leq n-k+1\} \cup\{(j+k-1, j): 1 \leq j \leq n-k+1\}$

which consists of positions in the upper right triangle and the lower left triangle.

Lemma 25. Each matrix $F_{n}^{k, r, s}$ is an $A S M$, and $\sigma_{-}\left(F_{n}^{k, r, s}\right)=(k-r-1)(n-$ $k)-s . F_{n}^{k, r, s}$ is obtained from $F_{n}^{k}$ by replacing some zeros in $K_{n, k}$ by 1 , and replacing some nonzeros outside $K_{n, k}$ by zero.

Proof. This follows from the construction of $F_{n}^{k, r, s}$.

Example 26. Let $n=8$ and $k=5$, so

$F_{8}^{5}=\left[\begin{array}{r|r|r|r|r|r|r|r} & & & & 1 & & & \\ \hline & & & 1 & -1 & 1 & & \\ \hline & & 1 & -1 & 1 & -1 & 1 & \\ \hline 1 & 1 & -1 & 1 & -1 & 1 & -1 & 1 \\ \hline & 1 & -1 & 1 & -1 & 1 & & \\ \hline & & 1 & -1 & 1 & & & \\ \hline & & & 1 & & & & \end{array}\right]$.


Then $p=n-k=3$ is odd. For $r=2$ and $s=0$, we obtain

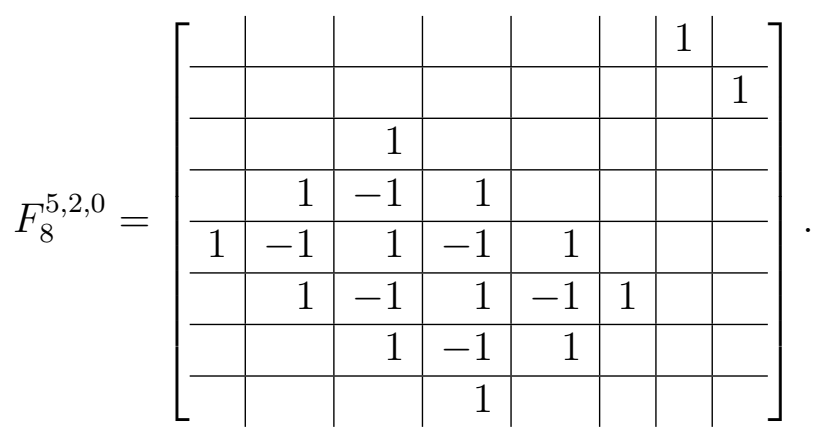

Here the first two positive and negative diagonals of $F_{n}^{k}$ now contain zeros, and, as a compensation, we have the two ones in the upper right corner. For $r=1$ and $s=2$, the first positive and negative diagonals are replaced by zeros, and a modification is done in the lower left corner:

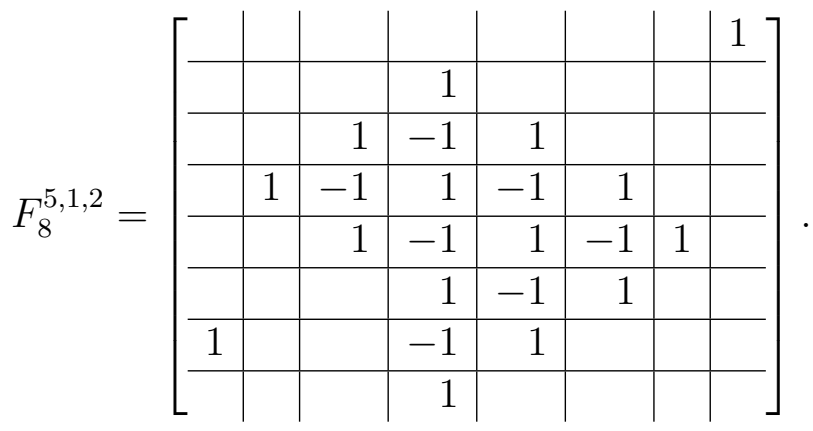

Theorem 27. Let $n$ be a positive integer. Then, where $m_{n}^{-}$is given by (15), for every integer $0 \leq t \leq m_{n}^{-}$, there exists an $A S H M A$ with $\sigma_{-}(A)=t$.

Proof. First, note that any permutation ASHM does not have any negative entries. Let $\mathfrak{D}_{n}=\left[A_{1}, A_{2}, \ldots, A_{n}\right]$ be the diamond ASHM of size $n \times n \times n$. Then $\mathfrak{D}_{n}$ has $m_{n}^{-}$negative entries. Let $1<t<m_{n}^{-}$. Choose $k$ minimal such that the hypermatrix $\left[A_{1}, A_{2}, \ldots, A_{k}\right]$ has at least $t$ negative entries. So $k \leq n-1$.

We claim that there exists an $n \times n$ ASM $B$ such that each vertical line in the $n \times n \times k$ hypermatrix $A^{\prime}=\left[A_{1}, A_{2}, \ldots, A_{k-1}, B\right]$ is $(1, *)$-alternating, i.e., (14) holds and, moreover, $A^{\prime}$ has $t$ negative entries.

The claim follows from Lemma 25 as we can choose $B=F_{n}^{k, r, s}$ for suitable $r$ and $s$ so that $B$ has $t$ negative entries (the $r$ and $s$ are unique). Then $B$ is 
an ASM and the $(1, *)$-alternating property follows from the second part of the lemma, as all the matrices $A_{1}, A_{2}, \ldots, A_{k-1}$ have only zeros in positions in $K_{n, k}$.

Now, due to the claim, we apply Theorem 23 to obtain $n \times n$ permutation matrices $P_{s}(k+1 \leq s \leq n)$ such that $A=\left[A_{1}, A_{2}, \ldots, A_{k-1}, B, P_{k+1}, P_{k+2}, \ldots, P_{n}\right]$ is an ASHM, and has exactly $t$ negative entries as desired.

We remark that the ASHM $A$ constructed in the proof of Theorem 27 is extreme in that it has the maximum number of elements in the first $k-1$ horizontal layers, and no negative entries in the last $n-k$ layers.

We now show that there exists an $n \times n \times n$ ASHM whose -1 's are confined to the $k$ th horizontal level and whose number of -1 's is any number below the maximum possible at the $k$ th horizontal level.

Theorem 28. Let $0 \leq t \leq \sigma_{-}\left(F_{n}^{k}\right)$. Then there exists an $n \times n$ ASM $A_{k}$ with $t$ negative entries and $n \times n$ permutation matrices $P_{1}, \ldots, P_{k-1}, P_{k+1}, \ldots, P_{n}$ such that

$$
A=\left[P_{1}, \ldots, P_{k-1}, A_{k}, P_{k+1}, \ldots, P_{n}\right]
$$

is an $n \times n \times n A S H M$.

Proof. We use the construction in the proof of Theorem 27. This shows that there is an ASM $A_{k}$ with $t$ negative entries and permutation matrices $P_{k+1}, \ldots, P_{n}$ such that $A^{\prime}=\left[F_{n}^{1}, \ldots, F_{n}^{k-1}, A_{k}, P_{k+1}, \ldots, P_{n}\right]$ is an ASHM.

Consider the subhypermatrix $A^{\prime \prime}=\left[A_{k}, P_{k+1}, \ldots, P_{n}\right]$ obtained from $A^{\prime}$ by deleting the first $k-1$ horizontal planes. Then, by reversing the order of these planes, each vertical line is $(1, *)$-alternating, so we apply Theorem 23 , and there exist $n \times n$ permutation matrices $P_{s}(1 \leq s \leq k-1)$ such that

$$
A=\left[P_{1}, P_{2}, \ldots, P_{k-1}, A_{k}, P_{k+1}, P_{k+2}, \ldots, P_{n}\right]
$$

is an ASHM, as desired.

Recall that a matrix is convex provided the nonzeros in each of its lines are consecutive. Examples of convex ASMs are the $n \times n$ ASMs $F_{n}^{k}$, the $k$ th horizontal ASM of the diamond ASHM $\mathfrak{D}_{n}$. An $n \times n \times n$ hypermatrix is convex provided the nonzeros in each of its three types of lines are consecutive. The diamond ASHM $\mathfrak{D}_{n}$ is convex. We call an ASM minus-convex provided in every row and column all entries between two negative entries (if any) are nonzero, i.e., each of its rows and columns has a form like

$$
0, \ldots, 0,1,0, \ldots, 0,-1,1,-1,1, \ldots,-1,0, \ldots, 0,1,0, \ldots, 0
$$


where the subsequences of zeros can be void.

Theorem 29. Let $B$ be an $n \times n$ matrix obtained from an $n \times n A S M A$ by replacing some nonzeros with zeros in such a way that the result is convex. Then $B$ can be completed to an $n \times n$ minus-convex $A S M B^{\prime}$ by changing some zeros to ones.

Before giving a proof of Theorem 29, which provides a simple algorithm to obtain $B^{\prime}$, we illustrate the theorem with an example.

Example 30. Consider the ASM

$$
A=F_{10}^{5}=\left[\begin{array}{r|r|r|r|r|r|r|r|r|r} 
& & & & 1 & & & & & \\
\hline & & & 1 & -1 & 1 & & & & \\
\hline & & 1 & -1 & 1 & -1 & 1 & & & \\
\hline & 1 & -1 & 1 & -1 & 1 & -1 & 1 & & \\
\hline 1 & -1 & 1 & -1 & 1 & -1 & 1 & -1 & 1 & \\
\hline & 1 & -1 & 1 & -1 & 1 & -1 & 1 & -1 & 1 \\
\hline & & 1 & -1 & 1 & -1 & 1 & -1 & 1 & \\
\hline & & & 1 & -1 & 1 & -1 & 1 & & \\
\hline & & & & 1 & -1 & 1 & & & \\
\hline & & & & & 1 & & & &
\end{array}\right]
$$

where the shaded entries are to be replaced with zeros to obtain a $10 \times 10$ convex matrix $B$. Then a completion of $B$ to an ASM obtained by changing certain zeros to ones is

$$
B^{\prime}=\left[\begin{array}{r|r|r|r|r|r|r|r|r|r} 
& & & & & 1 & & & & \\
\hline & & 1 & & & & & & & \\
\hline & & & & 1 & -1 & & 1 & & \\
\hline 1 & & -1 & 1 & -1 & 1 & & & & \\
\hline & & 1 & -1 & 1 & -1 & 1 & -1 & 1 & \\
\hline & 1 & -1 & 1 & -1 & 1 & -1 & 1 & -1 & 1 \\
\hline & & 1 & -1 & 1 & -1 & 1 & -1 & 1 & \\
\hline & & & 1 & -1 & 1 & -1 & 1 & & \\
\hline & & & & & & 1 & & &
\end{array}\right] .
$$

Proof. (of Theorem 29) If $B$ does not have any -1's (in particular, if $A$ does not have any -1 's), then $B$ consists of $p \geq 01$ 's no two on the same 
line, and $B$ can be completed to a permutation matrix and hence an ASM. We now assume that $B$ contains at least one -1 . To proceed we consider the following transformation of an ASM.

Let $A=\left[a_{i j}\right]$ be an ASM with at least one negative entry. Choose $(k, l)$ with $k+l$ minimal such that $a_{k l}=-1$. Then there is a unique $l^{\prime}<l$ with $a_{k l^{\prime}}$ nonzero, and we have $a_{k l^{\prime}}=1$. Similarly, there is a unique $k^{\prime}<k$ with $a_{k^{\prime} l}$ nonzero and $a_{k^{\prime} l}=1$. By the choice of $k, l$ there is no negative entry in the leading $k \times l$ submatrix, apart from $a_{k l}$. Moreover, $a_{k^{\prime} l^{\prime}}=0$. Let $C=\left[c_{i j}\right]$ be obtained from $A$ by letting $c_{k l}=c_{k^{\prime} l}=c_{k l^{\prime}}=0, c_{k^{\prime} l^{\prime}}=1$, and, otherwise, $c_{i j}=a_{i j}$. Then $C$ is an ASM and $\sigma_{-}(C)=\sigma_{-}(A)-1$. This transformation makes a change in the upper-left corner of the matrix $A$. Clearly a similar transformation may be performed in the upper-right, lower-left or lower-right corner of $A$. Each of these will be called a corner transform of an ASM.

Note that if $A$ is a minus-convex ASM and $C$ is obtained from $A$ by a corner transform, then $C$ is also minus-convex.

The theorem follows by a sequence of corner transforms: Let $A$ and $B$ be as stated in the theorem. If $B$ has a -1 wherever $A$ has a -1 , then we let $B^{\prime}=A$. Now assume that there is at least one position in which $A$ has a -1 and $B$ has a 0 . Choosing $(k, l)$ as above, we use a corner transformation and iteratively obtain a minus-convex ASM which has a -1 in every position that $B$ has a -1 . Proceeding inductively we obtain the desired ASM $B^{\prime}$.

Example 31. Let

$$
A=F_{5}^{3}=\left[\begin{array}{r|r|r|r|r} 
& & 1 & & \\
\hline & 1 & -1 & 1 & \\
\hline 1 & -1 & 1 & -1 & 1 \\
\hline & 1 & -1 & 1 & \\
\hline & & 1 & &
\end{array}\right]
$$

where the shaded entries are to be replaced with zeros to obtain a convex matrix $B$. Then using corner transformations we obtain an ASM:

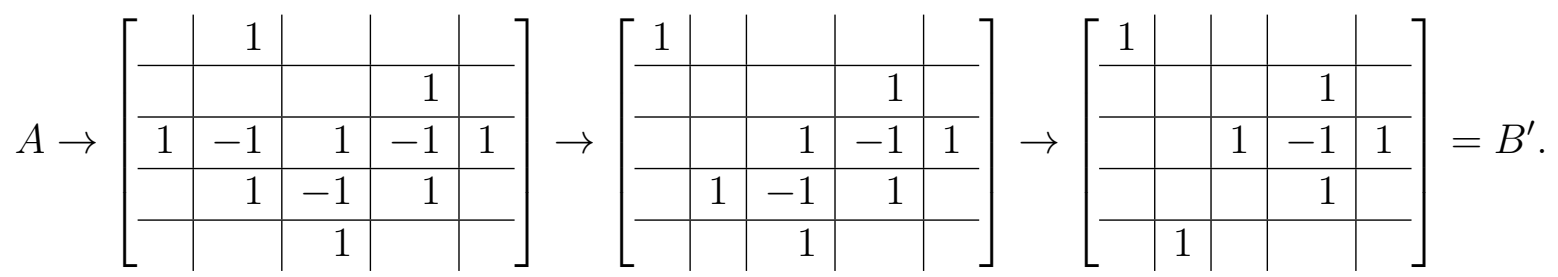


We now study another completion problem, where an ASM is given and we want to extend it to an ASHM in some way. First, we introduce a generalization of an ASM. Consider a $(0, \pm 1)$-matrix with all line sums equal to 1 ; such a matrix will be called a semi-ASM. Thus, if a line contains $p-1$ 's, then it has $(p+1)+1$ 's. If $A$ is a PASHM, then its row-vertical planes and column-vertical planes are semi-ASMs.

The semi-ASMs have the following simple property. With a semi-ASM $A=\left[a_{i j}\right]$ of order $n$ we associate the bipartite graph $G_{0}(A)$ of $A$ with $n$ vertices in each color class and having an edge $i j$ whenever $a_{i j}=0$; thus the edges correspond to the zeros and not, as is often the case, to the nonzeros. If $G_{0}(A)$ has a cycle (which must be even), then by putting +1 and -1 in the positions of $A$ of this cycle (alternating), we obtain a new matrix $B$ from $A$. Then $B$ is also a semi-ASM and has more nonzeros than $A$.

If $A$ and $B$ are semi-ASMs of order $n$ and $B$ agrees in every nonzero entry of $A$, we say that $B$ is a semi-ASM extension of $A$. Thus, the cycle construction above produces a semi-ASM extension $B$ of $A$, and we call this a cycle-extension.

Theorem 32. Let $A$ be a semi-ASM of order $n$ where $n$ is odd. Then $A$ has a semi-ASM extension $B$ with all entries nonzero.

Proof. The proof of this theorem uses a standard kind of argument. Assume $A$ has at least one zero entry (otherwise we are done). Let $L$ denote the set of lines in $A$ that contain at least one zero entry. Since $A$ is a semi-ASM, each line has an odd number of nonzero entries. Therefore, as $n$ is odd, each line in $L$ has a positive even number of zeros. Consider the subgraph of $G_{0}(A)$ induced by the vertices corresponding to $L$ (so we have just removed isolated vertices, that is, vertices corresponding to lines of all nonzeros). In this subgraph each vertex has an even, nonzero degree, and therefore the subgraph contains a cycle. Now, use this cycle and perform a cycle-extension of $A$. This gives a new matrix $A^{\prime}$ with fewer zeros than $A$, and $A^{\prime}$ is a semi-ASM. We may repeat this process, and get a sequence of semi-ASMs with fewer zeros, and eventually we obtain a semi-ASM $B$ with no zeros, as desired.

The next example shows that the property in Theorem 32 does not hold in general when $n$ is even. 
Example 33. Let $n=4$ and let the semi-ASM $A$ be given by

$$
A=\left[\begin{array}{rrrr}
0 & 0 & 0 & 1 \\
1 & -1 & 1 & 0 \\
1 & 1 & -1 & 0 \\
-1 & 1 & 1 & 0
\end{array}\right]
$$

To find an extension, in the first row, we need to change some 0 to -1 and another 0 to +1 , but then we violate that all column sums should be 1 . So a semi-ASM extension does not exist.

We say that two $n \times n(0,1)$-matrices $A_{1}$ and $A_{2}$ are disjoint provided that their supports are disjoint, that is, provided that $A_{1}+A_{2}$ is also a $(0,1)$-matrix.

Corollary 34. Let $n=2 k+1$ be odd, and let $A$ be a semi-ASM of order $n$. Then there is a decomposition of $J_{n}$ into pairwise disjoint permutation matrices $P_{1}, P_{2}, \ldots, P_{n}$ such that the ones in $P_{i}(i \leq k)$ cover all the -1 's in $A$, and the ones in $P_{i}(k<i \leq n)$ cover all the 1 's in $A$.

Proof. Apply Theorem 32 to $A$, and let $B$ be an extension with all entries nonzero. The $(0,1)$-matrix $B_{1}$ obtained from $B$ by replacing each 1 by 0 , and multiplying the matrix by -1 has all line sums equal to $k$, and can therefore be written as the sum of $k$ pairwise disjoint permutation matrices. Similarly, the $(0,1)$-matrix $B_{2}$ obtained from $B$ by replacing each -1 by 0 has all line sums equal to $k+1$, and can therefore be written as the sum of $k+1$ pairwise disjoint permutation matrices. These $n$ permutation matrices are pairwise disjoint, so the result follows.

We now use the previous results to solve the completion problem mentioned above, where we want to extend a given ASM into an ASHM.

Theorem 35. Let $B$ be an ASM of order $n$ where $n=2 k+1$ is odd. Then there are $n-1$ permutation matrices $P_{i}(i \leq n, i \neq k)$ such that

$$
A=\left[P_{1}, \ldots, P_{k}, B, P_{k+1}, \ldots, P_{n}\right]
$$

is an $A S H M$.

Proof. First, by Corollary 34 there exist pairwise disjoint permutation matrices $P_{1}, P_{2}, \ldots, P_{k}$ whose ones cover all the -1 's in $B$, but do not cover any of the 1 's in $B$. Therefore the matrix

$$
C=P_{1}+\cdots+P_{k}+B
$$


is a $(0,1)$-matrix. Consider the $n \times n \times(k+1)$ hypermatrix $A^{\prime}=\left[a_{i j s}\right]=$ $\left[P_{1}, \ldots, P_{k}, B\right]$. As $C$ is a $(0,1)$ matrix and the $P_{i}$ 's are pairwise disjoint, each of the vertical lines $\left(a_{i j 1}, \ldots, a_{i j, k+1}\right)$ is alternating $(i, j \leq n)$. So, (14) holds with $k$ replaced by $k+1$, and by Theorem 23 there are permutation matrices $P_{k+1}, P_{k+2}, \ldots, P_{n}$ such that $A=\left[P_{1}, \ldots, P_{k}, B, P_{k+1}, \ldots, P_{n}\right]$ is an ASHM.

Example 36. Let $n=3$ and

$$
B=\left[\begin{array}{rrr}
0 & 1 & 0 \\
1 & -1 & 1 \\
0 & 1 & 0
\end{array}\right]
$$

Then, in Theorem 35, we may let $P_{1}$ be the identity matrix (it covers the -1 , but none of the ones in $B$ ), and this gives the following ASHM with $B$ as the middle plane:

$$
A:=\left[\begin{array}{lll}
1 & 0 & 0 \\
0 & 1 & 0 \\
0 & 0 & 1
\end{array}\right] \nearrow\left[\begin{array}{rrr}
0 & 1 & 0 \\
1 & -1 & 1 \\
0 & 1 & 0
\end{array}\right] \nearrow\left[\begin{array}{lll}
0 & 0 & 1 \\
0 & 1 & 0 \\
1 & 0 & 0
\end{array}\right] .
$$

We now consider the case of even $n$. First we make a general definition. Let $B_{1}$ and $B_{2}$ be $n \times n(0,1,-1)$-matrices. We define $B_{1}$ and $B_{2}$ to be sign-disjoint provided $B_{1}+B_{2}$ is also a $(0,1,-1)$-matrix. Thus $B_{1}$ and $B_{2}$ are sign-disjoint if and only if they have neither 1 's in the same position nor -1 's in the same position. If $B_{1}$ and $B_{2}$ are $(0,1)$-matrices, then $B_{1}$ and $B_{2}$ are sign-disjoint if and only if they are disjoint as previously defined. We also observe that if $A=\left[A_{1}, A_{2}, \ldots, A_{n}\right]$ is an $n \times n \times n$ ASHM, then $A_{i}$ and $A_{i+1}$ are sign-disjoint for each $i=1,2, \ldots, n-1$.

Lemma 37. Let $n$ be an even integer, and let $B_{1}$ and $B_{2}$ be $n \times n$ signdisjoint ASMs. Then the line sums of $B_{1}+B_{2}$ all equal 2, and the number of zeros in each row and column of $B_{1}+B_{2}$ is even.

Proof. Since $B_{1}$ and $B_{2}$ are ASMs, all of their line sums equal 1, and hence all of the line sums of $B_{1}+B_{2}$ equal 2. Also, since $B_{1}$ and $B_{2}$ are ASMs, each contains an odd number of nonzeros in each row and column. Consider some row (or column) $i$ of $B_{1}$ and $B_{2}$. Let the number of nonzero positions 
in row $i$ of $B_{1}$ (respectively, $B_{2}$ ) be $n_{1}$ (respectively, $n_{2}$ ), and let $c$ be the number of positions in row $i$ in which $B_{1}$ has a 1 and $B_{2}$ has a -1 or the other way around. Then the number of nonzeros in row $i$ of $B_{1}+B_{2}$ equals $n_{1}+n_{2}-2 c$, an even number. Since $n$ is also even, the number of zeros in row $i$ of $B_{1}+B_{2}$ is even.

Corollary 38. Let $n$ be an even integer, and let $B_{1}$ and $B_{2}$ be $n \times n$ signdisjoint ASMs. Then there is a $(0,1,-1)$-matrix $B$ which extends $B_{1}+B_{2}$ with all entries nonzero and all line sums equal to 2 . Moreover, there are pairwise disjoint permutation matrices $P_{1}, P_{2}, \ldots, P_{n}$ such that the permutation matrices $P_{1}, \ldots, P_{\frac{n}{2}-1}$ cover all the -1 's of $B_{1}+B_{2}$, and the permutation matrices $P_{\frac{n}{2}}, \ldots, P_{n}$ cover all the 1 's of $B_{1}+B_{2}$.

Proof. The proof follows as in the proofs of Theorem 32 and Corollary 34.

Theorem 39. Let $B_{1}$ and $B_{2}$ be $n \times n$ sign-disjoint $A S M s$ where $n=2 k$ is even. Then there are $n-2$ permutation matrices $P_{i}\left(1 \leq i \leq n, i \neq \frac{n}{2}, \frac{n}{2}+1\right)$ such that

$$
A=\left[P_{1}, \ldots, P_{\frac{n}{2}-1}, B_{1}, B_{2}, P_{\frac{n}{2}+2}, \ldots, P_{n}\right]
$$

is an $A S H M$.

Proof. By Corollary 38, there is a $(0,1,-1)$-matrix $B$ which extends $B_{1}+$ $B_{2}$ with all entries nonzero and all line sums equal to 2 . Let $D$ be the $(0,1)$ matrix obtained from $B$ by replacing each 1 with a 0 and multiplying the matrix by -1 . Then all line sums of $D$ equal $\frac{n}{2}-1$, and the matrix $P_{1}+\cdots+$ $P_{\frac{n}{2}-1}$ is a $(0,1)$-matrix. As in the proof of Theorem 35 there are permutation matrices $P_{\frac{n}{2}+2}, \ldots, P_{n}$ such that $A=\left[P_{1}, \ldots, P_{\frac{n}{2}-1}, B_{1}, B_{2}, P_{\frac{n}{2}+2}, \ldots, P_{n}\right]$ is an ASHM.

Let $n \geq 2$ be an integer and let $A$ be an $n \times n$ ASM. An $n \times n$ ASM $B$ is an $A S M$-mate of $A$ provided that $A+B$ is a $(0,1,-1)$-matrix, that is, provided that $A$ and $B$ are sign-disjoint.

Theorem 40. Let $A$ be an $A S M$ of order $n$. Then $A$ has an ASM-mate $B$ which may be chosen as a permutation matrix.

Proof. First, assume $n$ is odd, say $n=2 k+1$. By Corollary 34 there are pairwise disjoint permutation matrices $P_{1}, P_{2}, \ldots, P_{n}$ such that the ones in $P_{i}(i \leq k)$ cover all the -1 's in $A$, and the ones in $P_{i}(k<i \leq n)$ cover all 
the 1 's in $A$. In particular, $P_{1}$ covers only entries that are -1 or 0 (by the pairwise disjointness, and as the ones are covered by other $P_{i}$ 's). Therefore $A+P_{1}$ is a $(0,1,-1)$-matrix, and $P_{1}$ is an ASM-mate of $A$.

Next, assume $n$ is even, say $n=2 k$, and let $A=\left[a_{i j}\right]$. Let $I$ be a nonempty subset of $\{1,2, \ldots, n\}$, and let

$$
J_{I}=\left\{j \leq n: a_{i j} \in\{0,-1\} \text { for at least one } i \in I\right\} .
$$

If $|I| \leq k$, then $\left|J_{I}\right| \geq k \geq|I|$, since every row in $A$ has at most $k 1$ 's (as $A$ is an ASM) and therefore at least $k$ entries in $\{0,-1\}$. Otherwise, $|I|>k$, and then every column in $A$ must contain a 0 or a -1 in some row in $I$, as every column of $A$ contains at most $k 1$ 's. So, then $\left|J_{I}\right|=n \geq|I|$.

This proves that $\left|J_{I}\right| \geq|I|$ for every nonempty subset $I$ of $\{1,2, \ldots, n\}$, and by Hall's (marriage) theorem, there must be a permutation matrix $P$ whose ones are in the positions where $A$ has entries in $\{0,-1\}$. Therefore $A+P$ is a $(0,1,-1)$-matrix, and $P$ is an ASM-mate of $A$.

By definition, if we delete planes in a hypermatrix, we obtain a subhypermatrix. We next show that any $(0, \pm 1)$-hypermatrix is the subhypermatrix of some ASHM. This result is useful for constructing classes of ("random") ASHMs. A similar result for ASM submatrices was established in [5].

Let $A=\left[A_{1}, A_{2}, \ldots, A_{p}\right]=\left[a_{i j k}\right]$ be an $m \times n \times p$ hypermatrix where $A_{1}, A_{2}, \ldots, A_{p}$ are the horizontal planes of $A$. Let $k<p$. Define the $(0, \pm 1)$ matrix $C^{A, k}=\left[c_{i j}\right]$ of size $m \times n$ as follows. Let $i \leq m$ and $j \leq n$. If the last nonzero, if any, in the (partial) vertical line $\left(a_{i j 1}, a_{i j 2}, \ldots, a_{i j, k-1}\right)$ is -1 (resp. 1) and $a_{i j k}=-1$ (resp. 1), we define $c_{i j}=1$ (resp. $c_{i j}=-1$ ). All other entries of $C$ are zero. The idea of the construction algorithm is to insert planes between $A_{k-1}$ and $A_{k}$ based on the matrix $C^{A, k}$. The following result will be useful, see [8]. A subpermutation matrix is a $(0,1)$-matrix with at most one 1 in every row and column.

Theorem 41. ([8]) Let $A$ be a $(0,1)$-matrix with maximum line sum $t$. Then there are $t$ pairwise disjoint subpermutation matrices $P_{1}, P_{2}, \ldots, P_{t}$ such that

$$
A=P_{1}+P_{2}+\cdots+P_{t} .
$$

We now formulate the announced result.

Theorem 42. Let $A^{\prime}$ be an $(0, \pm 1)$-hypermatrix of size $m^{\prime} \times n^{\prime} \times k^{\prime}$. Then there is an integer $n \geq m^{\prime}, n^{\prime}, k^{\prime}$ and an $n \times n \times n A S H M A$ with $A^{\prime}$ as a subhypermatrix. In fact, $A$ may be obtained from $A^{\prime}$ by repeatedly inserting new 
planes (horizontal, column-vertical and row-vertical) that are subpermutation matrices and their negatives.

Proof. Let $A^{\prime}=\left[A_{1}, A_{2}, \ldots, A_{k^{\prime}}\right]=\left[a_{i j k}\right]$. We describe an algorithm for constructing the desired ASHM $A$. Start with the given $A^{\prime}$, and let $t_{0}$ be the maximum number of -1 's in a line of $A_{1}$ (so $t_{0} \geq 0$ ). By Theorem 41 there are pairwise disjoint $m^{\prime} \times n^{\prime}$ subpermutation matrices $P_{1}^{1}, P_{2}^{1}, \ldots, P_{t_{0}}^{1}$ such that the union of their supports coincides with the set of positions of the -1 's in $A_{1}$. Let

$$
A=\left[P_{1}^{1}, P_{2}^{1}, \ldots, P_{t_{0}}^{1}, A_{1}, A_{2}, \ldots, A_{k^{\prime}} .\right.
$$

Therefore each vertical line of the subhypermatrix

$$
\bar{A}=\left[P_{1}^{1}, P_{2}^{1}, \ldots, P_{t_{0}}^{1}, A_{1}\right]
$$

of $A$ is $(1, *)$-alternating (which includes the possibility of the zero vector). Next, if needed, we modify $A$ by adding horizontal planes between $A_{1}$ and $A_{2}$. Let $C=C^{A^{\prime}, t_{0}+2}$. Let $t_{1}$ (resp. $s_{1}$ ) be the maximum number of 1 's (resp. - 1's) in a line of $C$. By Theorem 41, there are pairwise disjoint subpermutation matrices $P_{1}^{2}, P_{2}^{2}, \ldots, P_{t_{1}}^{2}$ (resp. $Q_{1}^{2}, Q_{2}^{2}, \ldots, Q_{s_{1}}^{2}$ ) such that the union of their supports coincides with the set of positions of the 1's (resp. the -1 's) in $C$, and, moreover, $P_{i}^{2}$ and $Q_{j}^{2}$ are pairwise disjoint for each $i, j$. Then we update $A$ so that

$$
A=\left[P_{1}^{1}, P_{2}^{1}, \ldots, P_{t_{0}}^{1}, A_{1}, P_{1}^{2}, P_{2}^{2}, \ldots, P_{t_{1}}^{2},-Q_{1}^{2},-Q_{2}^{2}, \ldots,-Q_{s_{1}}^{2}, A_{2}, \ldots, A_{k^{\prime}}\right] .
$$

Then, by construction, each vertical line in $A$ up to, and including, the matrix $A_{2}$ is $(1, *)$-alternating. We continue in the same way, by inserting planes that are subpermutation matrices or their negatives between $A_{i}$ and $A_{i+1}$, so that the resulting matrix $A$ has $(1, *)$-alternating vertical lines up to its last plane $A_{k^{\prime}}$. Then we identify those $(i, j)$ for which the vertical line $(i, j, *)$ misses a final 1 , to make the line alternating, and add planes as above to achieve that. As a result, each vertical line in $A$ is alternating (and, therefore, nonzero).

The next step is to consider the column-vertical planes $A_{i * *}$ in $A$. We repeat the procedure above by inserting suitable column-vertical planes so that new hypermatrix $A$ has alternating row lines. Note that these plane insertions do not affect the alternating property of the vertical lines because every vertical line is included in (exactly) one column-vertical plane. 
Finally, we repeat the procedure another time, now for the columnvertical planes $A_{* j *}$ in $A$. Again, the previous alternating properties are maintained. The resulting $m \times n \times k$ matrix $A$ has only alternating lines in each direction. Since every line sum in $A$ is 1 , summation shows that $m=n=k$, so $A$ is an ASHM as desired.

Finally we mention that a different kind of ASM completion problem was considered in $[6,7]$.

\section{Coda}

As a generalization of alternating sign matrices and Latin squares, we have initiated the study of the fascinating class of ASHMs and the associated class of ASHM Latin-like squares. In view of the results obtained, there are many possible directions to pursue. In particular, a characterization of ASHLs would be very interesting. A specific question is the following.

Problem 43. What is the maximum number of times an integer can occur as an entry in an $n \times n$ ASHL? The $7 \times 7$ ASHL in (10) and the subsequent discussion shows that an integer can occur $2 n$ times in an $n \times n$ ASHL. Is the maximum equal to $2 n$ ?

As a step in characterizing the entries of an ASHL, we would like to characterize the weighted projections of ASMs.

Conjecture 44. Let $\left(c_{1}, c_{2}, \ldots, c_{n}\right)$ be a vector of positive integers such that $\left(c_{1}, c_{2}, \ldots, c_{n}\right) \preceq(n, n-1, \ldots, 2,1)$. Then there exists an $n \times n$ ASM whose weighted vertical projection is $\left(c_{1}, c_{2}, \ldots, c_{n}\right)$.

Another possible and worthwhile direction is provided by the concept of orthogonality of Latin squares which is fundamentally important and wellstudied in combinatorics.

Let

$$
L_{1}=1 P_{1}+2 P_{2}+\cdots+n P_{n} \text { and } L_{2}=1 Q_{1}+2 Q_{2}+\cdots+n Q_{n}
$$

be two $n \times n$ Latin squares, where $P_{1}, P_{2}, \ldots, P_{n}$ and $Q_{1}, Q_{2}, \ldots, Q_{n}$ are $n \times n$ permutation matrices with $P_{1}+P_{2}+\cdots+P_{n}=J_{n}$ and $Q_{1}+Q_{2}+\cdots+Q_{n}=J_{n}$. Then $L_{1}$ and $L_{2}$ are orthogonal provided when they are juxtaposed to form a matrix $L_{1} * L_{2}$ of ordered pairs, all pairs $(i, j)$ with $1 \leq i, j \leq n$ occur 
(equivalently, there are no repeats); $L_{1}$ and $L_{2}$ are then called orthogonal $L S$-mates. If $n>2$ but $n \neq 6$, the celebrated theorem of Bose, Parker, and Shrikhande (see [14] for a discussion and more references) asserts that there exists a pair of orthogonal $n \times n$ Latin squares. It is well-known that for each $n \geq 2$, the maximum number of pairwise orthogonal Latin squares is bounded by $(n-1)$.

Another way to view orthogonality of Latin squares is the following: The $n \times n$ Latin squares $L_{1}$ and $L_{2}$ in (16) are orthogonal if and only if the standard inner products (sum of products of corresponding entries) of the $P_{i}$ and the $Q_{j}$ satisfy

$$
\left\langle P_{i}, Q_{j}\right\rangle=1 \quad(1 \leq i, j \leq n),
$$

equivalently, each pair $P_{i}$ and $Q_{j}$ have exactly one 1 in common positions. Let us define $P_{i}$ and $Q_{j}$ to be orthogonal permutation-mates when $\left\langle P_{i}, Q_{j}\right\rangle=1$. Thus the Latin squares $L_{1}$ and $L_{2}$ in (16) are orthogonal LS-mates if and only if $P_{i}$ and $Q_{j}$ are orthogonal permutation-mates for all $i$ and $j$. If $n \geq 2$, every $n \times n$ permutation matrix has an orthogonal permutation-mate (in fact, exactly $n D_{n-1}$ orthogonal mates where $D_{n-1}$ is the $(n-1)$ st derangement number). But, as is well known, not every Latin square has an orthogonal LS-mate.

Now consider two ASHLs.

$$
L(A)=1 A_{1}+2 A_{2}+\cdots+n A_{n} \text { and } L(B)=1 B_{1}+2 B_{2}+\cdots+n B_{n},
$$

where $A=\left[A_{1}, A_{2}, \ldots, A_{n}\right]$ and $B=\left[B_{1}, B_{2}, \ldots, B_{n}\right]$ are $n \times n \times n$ ASHMs. Thus

$$
J_{n}=A_{1}+A_{2}+\cdots+A_{n} \text { and } J_{n}=B_{1}+B_{2}+\cdots+B_{n} .
$$

Generalizing orthogonality of Latin squares, with the $A_{i}$ and $B_{j}$ playing the role of the permutation matrices $P_{i}$ and $Q_{j}$ above, we define the ASHLs $L(A)$ and $L(B)$ to be orthogonal provided the inner products of the $A_{i}$ with the $B_{j}$ satisfy

$$
\left\langle A_{i}, B_{j}\right\rangle=1 \quad(1 \leq i, j \leq n) .
$$

If $L(A)$ and $L(B)$ are orthogonal, then we also say that $L(A)$ and $L(B)$ are orthogonal ASHL mates. It follows from the theorem of Bose, Parker, and Shrikhande that, if $n>2$ but $n \neq 6$, then there exists a pair of orthogonal ASHLs. Extending our definition above from permutation matrices to ASHMs, we define the ASMs $A_{i}$ and $B_{j}$ to be orthogonal ASM-mates when $\left\langle A_{i}, B_{j}\right\rangle=1$. 
Example 45. The ASM

$$
\left[\begin{array}{rrr}
0 & 1 & 0 \\
1 & -1 & 1 \\
0 & 1 & 0
\end{array}\right]
$$

does not have an orthogonal ASM-mate. The ASMs

$$
\left[\begin{array}{rrrrr}
0 & 0 & 1 & 0 & 0 \\
0 & 1 & -1 & 1 & 0 \\
1 & -1 & 1 & -1 & 1 \\
0 & 1 & -1 & 1 & 0 \\
0 & 0 & 1 & 0 & 0
\end{array}\right],\left[\begin{array}{lllll}
0 & 1 & 0 & 0 & 0 \\
1 & 0 & 0 & 0 & 0 \\
0 & 0 & 1 & 0 & 0 \\
0 & 0 & 0 & 0 & 1 \\
0 & 0 & 0 & 1 & 0
\end{array}\right] \text {, and }\left[\begin{array}{lllll}
0 & 0 & 0 & 1 & 0 \\
0 & 0 & 0 & 0 & 1 \\
0 & 0 & 1 & 0 & 0 \\
1 & 0 & 0 & 0 & 0 \\
0 & 1 & 0 & 0 & 0
\end{array}\right]
$$

are pairwise orthogonal ASM-mates.

This extension of the notion of orthogonality leads to some questions

Problem 46. (a) Which $n \times n$ ASMs have an orthogonal ASM-mate? Which $n \times n$ ASMs have a permutation matrix as an orthogonal mate?

(b) Does there exist a set of more than $(n-1)$ pairwise orthogonal $n \times n \times n$ ASHLs?

One possible way to consider the question (b) is to start with a set of $(n-1)$ pairwise orthogonal $n \times n$ Latin squares (so coming from $(n-1)$ $n \times n \times n$ permutation ASHMs), and construct an $n \times n \times n$ ASHL orthogonal to each of them. This possibility addresses the question as to whether or not there exists a set of $n$ pairwise orthogonal ASHLs extending a set of $(n-1)$ pairwise orthogonal Latin squares.

Since there does not exist a pair of $6 \times 6$ orthogonal Latin squares and since orthogonal ASHLs are less restrictive than orthogonal Latin squares, in the original version of this paper (posted on the arXiv) we raised the question of the existence of a pair of orthogonal $6 \times 6$ ASHLs. Through computation, Eric Egge found several such pairs. We are grateful to him for allowing us to include one such pair in the next example.

Example 47. Consider the two ASHMs $A=\left[A_{1}, A_{2}, A_{3}, A_{4}, A_{5}, A_{6}\right]$ and 
$B=\left[B_{1}, B_{2}, B_{3}, B_{4}, B_{5}, B_{6}\right]$ defined by
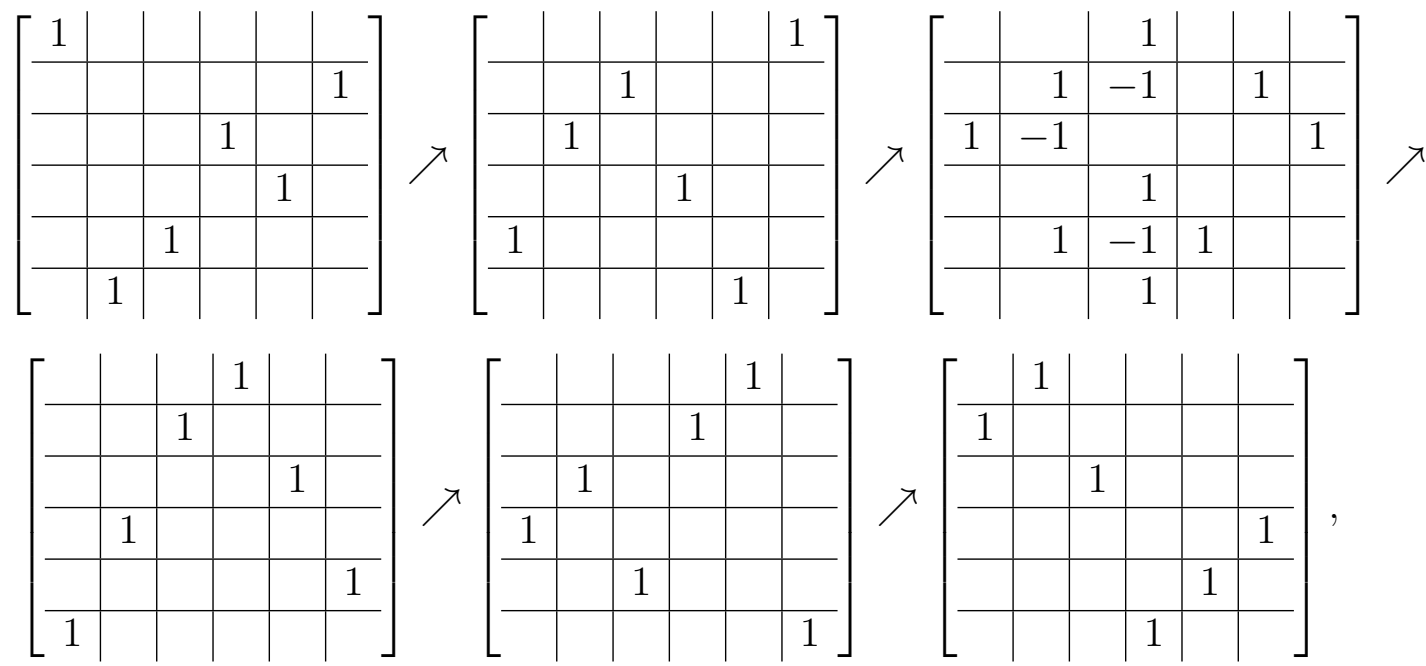

and
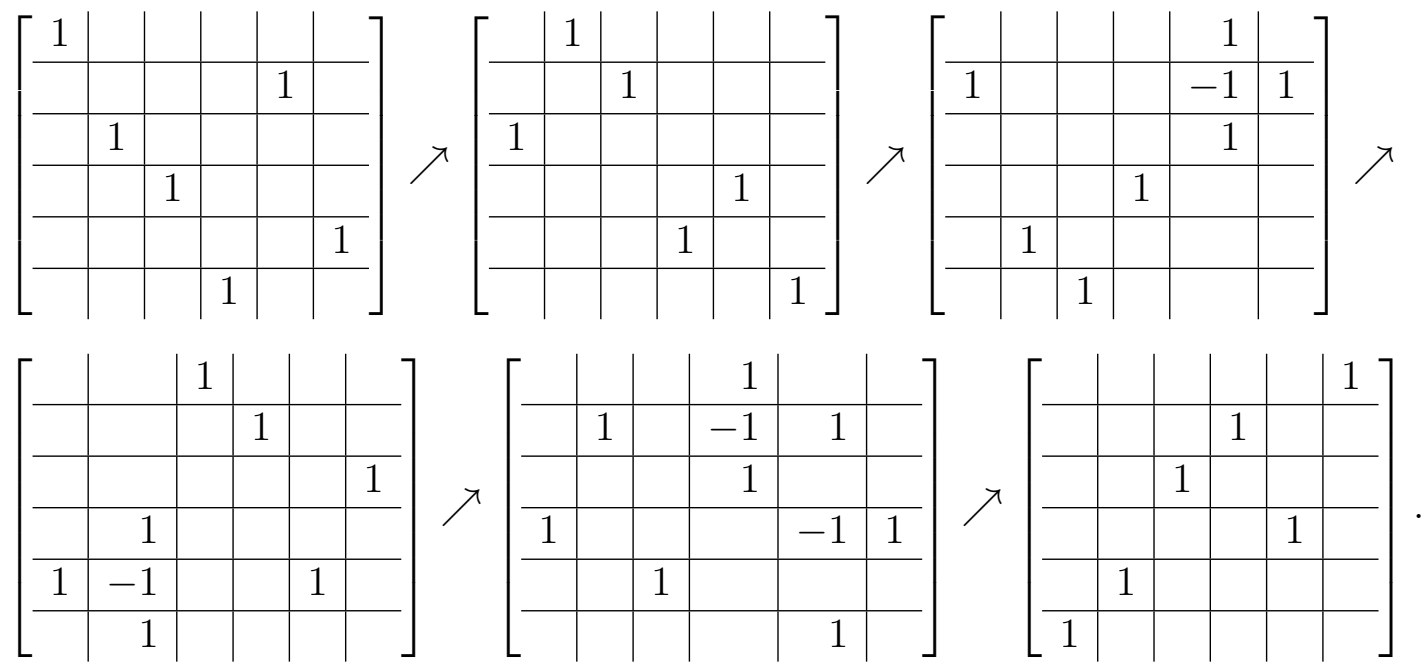

It is easily checked that $\left\langle A_{i}, B_{j}\right\rangle=1$ for $1 \leq i, j \leq 6$, and $A$ and $B$ give the following pair of orthogonal $6 \times 6$ ASHLs:

$$
L(A)=\left[\begin{array}{llllll}
1 & 6 & 3 & 4 & 5 & 2 \\
6 & 3 & 3 & 5 & 3 & 1 \\
3 & 4 & 6 & 1 & 4 & 3 \\
5 & 4 & 3 & 2 & 1 & 6 \\
2 & 3 & 3 & 3 & 6 & 4 \\
4 & 1 & 3 & 6 & 2 & 5
\end{array}\right] \text { and } L(B)=\left[\begin{array}{cccccc}
1 & 2 & 4 & 5 & 3 & 6 \\
3 & 5 & 2 & 5 & 3 & 3 \\
2 & 1 & 6 & 5 & 3 & 4 \\
5 & 4 & 1 & 3 & 3 & 5 \\
4 & 5 & 5 & 2 & 4 & 1 \\
6 & 4 & 3 & 1 & 5 & 2
\end{array}\right] .
$$


If $L$ is an $n \times n$ Latin square, then $L$ corresponds to a unique $n$-tuple $\left(P_{1}, P_{2}, \ldots, P_{n}\right)$ of $n \times n$ permutation matrices with $J_{n}=P_{1}+P_{2}+\cdots+P_{n}$, that is, to a unique $n \times n \times n$ permutation hypermatrix $\left[P_{1}, P_{2}, \ldots, P_{n}\right]$, such that $L=1 P_{1}+2 P_{2}+\cdots+n P_{n}$. This leads to the following question for ASHLs.

Problem 48. Given an $n \times n$ ASHL $L$, let $\mathcal{A}_{n}(L)$ be the set of all $n \times n \times n$ ASHMs $A$ such that $L(A)=L$. Investigate $\mathcal{A}_{n}(L)$. Note that if $L$ is an ordinary Latin square, then $\mathcal{A}_{n}(L)$ contains a unique ASHM $A$ and $A$ is a permutation hypermatrix.

Acknowledgment. We thank a referee for several useful comments.

\section{References}

[1] R.E. Behrend, V.A. Knight, Higher spin alternating sign matrices, Electron. J. Combin. 14 (2007), 1-38.

[2] D. Bressoud, Proofs and Confirmations: The Story of the Alternating Sign Matrix Conjecture, Math. Association of America, Cambridge University Press 1999.

[3] R.A. Brualdi, Combinatorial Matrix Classes, Cambridge University Press, Cambridge, 2006.

[4] R.A. Brualdi, G. Dahl, Alternating sign matrices, extensions and related cones, Advances in Applied Mathematics, 86 (2017), 19-49.

[5] R.A. Brualdi, K.P. Kiernan, S.A. Meyer, M.S. Schroeder, Patterns of Alternation Sign Matrices, Linear Alg. Appl. 438 (2013), 3967-3990.

[6] R.A. Brualdi, H.-K. Kim, Symmetric Alternating Sign Matrices, Australasian J. Combinatorics, 60 (2014), 333-345.

[7] R.A. Brualdi, H.-K. Kim, Completions of Alternating Sign Matrices, Graphs $\& 3$ Combinatorics, 31 (2015), 507-522. 
[8] R.A. Brualdi, H.J. Ryser, Combinatorial Matrix Theory, Cambridge University Press, Cambridge, 1991.

[9] R.A. Brualdi, M.W. Schroeder, Alternating sign matrices and their Bruhat order, Discrete Math., 340 (2017), 1996-2019.

[10] J. Csima, Multidimensional stochastic matrices and patterns, J. Algebra, 14 (1970), 194-202.

[11] L. Cui, W. Li., M. Ng, Birkhoff-von Neumann theorem for multistochastic tensors, SIAM J. Matrix Anal. Appl., 35 (2014), No. 3, 956-973.

[12] M. R. Garey, D. S. Johnson, Computers and Intractability: A Guide to the Theory of NP-completeness, W. H. Freeman, New York, 1979.

[13] W.H. Mills, D.P. Robbins, H. Rumsey, Alternating-sign matrices and descending plane partitions, J. Combin. Theory Ser. A, 34 (1983), 340359.

[14] H.J. Ryser, Combinatorial Mathematics, Carus Math. Monograph, No. 14, Math. Assoc. of America, Providence, 1963.

[15] J. Striker, The alternating sign matrix polytope, Electron. J. Combin., 16, \#R41 (2009). 